\title{
OPTIMAL CONTROL FOR THE THERMISTOR PROBLEM*
}

\author{
D. HÖMBERG ${ }^{\dagger}$, C. MEYER ${ }^{\dagger}$, J. REHBERG ${ }^{\dagger}$, AND W. RING $^{\ddagger}$
}

\begin{abstract}
This paper is concerned with the state-constrained optimal control of the twodimensional thermistor problem, a quasi-linear coupled system of a parabolic and elliptic PDE with mixed boundary conditions. This system models the heating of a conducting material by means of direct current. Existence, uniqueness, and continuity for the state system are derived by employing maximal elliptic and parabolic regularity. By similar arguments the linearized state system is discussed, while the adjoint system involving measures is investigated using a duality argument. These results allow us to derive first-order necessary conditions for the optimal control problem.
\end{abstract}

Key words. partial differential equations, optimal control problems, state constraints

AMS subject classifications. 35K55, 35M10, 49J20, 49K20

DOI. $10.1137 / 080736259$

1. Introduction. In this paper we consider state-constrained optimal control of the two-dimensional thermistor problem. In detail the optimal control problem under consideration looks as follows:

$$
\begin{aligned}
& \text { minimize } \quad J(\theta, \varphi, u):=\frac{1}{2} \int_{D}\left|\theta(T)-\theta_{d}\right|^{2} d x+\frac{\beta}{2} \int_{\Sigma_{N}} u^{2} d s d t \\
& \text { subject to (1.1)-(1.7) } \\
& \text { and } \quad \theta(x, t) \leq \theta_{\max }(x, t) \text { a.e. in } Q \text {, } \\
& 0 \leq u(x, t) \leq u_{\max }(x, t) \quad \text { a.e. on } \Sigma_{N}, \\
& \}
\end{aligned}
$$

where (1.1)-(1.7) refer to the following coupled PDE system consisting of the instationary heat equation and the quasi-static potential equation, which is also known as the thermistor problem:

$$
\begin{aligned}
\partial_{t} \theta-\operatorname{div}(\kappa \nabla \theta) & =(\sigma(\theta) \nabla \varphi) \cdot \nabla \varphi & & \text { in } Q:=\Omega \times] 0, T[, \\
\nu \cdot \kappa \nabla \theta+\alpha \theta & =\alpha \theta_{l} & & \text { on } \Sigma:=\partial \Omega \times] 0, T[, \\
\theta(0) & =\theta_{0} & & \text { in } \Omega, \\
-\operatorname{div}(\sigma(\theta) \nabla \varphi) & =0 & & \text { in } Q, \\
\nu \cdot \sigma(\theta) \nabla \varphi & =u & & \text { on } \left.\Sigma_{N}:=\Gamma_{N} \times\right] 0, T[, \\
\varphi & =0 & & \text { on } \left.\Sigma_{D}:=\Gamma_{D} \times\right] 0, T[, \\
\nu \cdot \sigma(\theta) \nabla \varphi & =0 & & \text { on } \left.\left(\partial \Omega \backslash \bar{\Gamma}_{N} \cup \Gamma_{D}\right) \times\right] 0, T[.
\end{aligned}
$$

Here $\theta$ is the temperature in a conducting material covered by the two-dimensional domain $\Omega$, while $\varphi$ refers to the electric potential. The boundary of $\Omega$ is denoted by $\partial \Omega$ with unit normal $\nu$ facing outward from $\Omega$. In addition, $\Gamma_{D}$ is a closed part of

\footnotetext{
${ }^{*}$ Received by the editors September 24, 2008; accepted for publication (in revised form) October 30, 2009; published electronically February 17, 2010.

http://www.siam.org/journals/sicon/48-5/73625.html

${ }^{\dagger}$ Weierstrass Institute for Applied Analysis and Stochastics, Mohrenstr. 39, D-10117 Berlin, Germany (hoemberg@wias-berlin.de, cmeyer@gsc.tu-darmstadt.de, rehberg@wias-berlin.de).

$\ddagger$ Institute of Mathematics, University of Graz, Heinrichstr. 36, A-8010 Graz, Austria (wolfgang. ring@uni-graz.at).
} 
$\partial \Omega$, while $\Gamma_{N}$ is an open part of $\partial \Omega$ which is disjoint to $\Gamma_{D}$. Moreover, $T$ is a given end time, $Q=\Omega \times] 0, T[$ is the space-time cylinder with boundary $\Sigma=\partial \Omega \times] 0, T[$, and $\Sigma_{N}$ and $\Sigma_{D}$ are defined analogously. Furthermore, $\kappa$ and $\sigma$ represent heat and electric conductivity. While $\kappa$ is a given prescribed function, $\sigma$ is allowed to depend on the temperature. Moreover, $\alpha$ is the heat transfer coefficient, and $\theta_{l}$ and $\theta_{0}$ are given boundary and initial data, respectively. The bounds in the optimization problem (P) as well as the desired temperature $\theta_{d}$ are given functions, and $\beta$ is the usual Tikhonov regularization parameter. Finally, $D$ is an open part of $\Omega$, and $u$ denotes the control. The precise assumptions on the data in (P) and (1.1)-(1.7) will be specified in section 2. In all of what follows, the system (1.1)-(1.7) is frequently also called a state system.

The PDE system (1.1)-(1.7) models the heating of a conducting material by means of a direct current induced on the part $\Gamma_{N}$ of the boundary. At the anode $\Gamma_{D}$, homogeneous Dirichlet boundary conditions are given, whereas one has insulation on $\partial \Omega \backslash \bar{\Gamma}_{N} \cup \Gamma_{D}$. We point out that the different boundary conditions are essential for a realistic modeling of the process. The objective of $(\mathrm{P})$ is to adjust the induced current $u$ to minimize the $L^{2}$-distance between the desired and the induced temperatures at end time $T$. Moreover, the optimization is subject to pointwise control and state constraints. The control constraints reflect a maximum heating power, while the state constraints limit the temperature evolution to prevent possible damage, e.g., by melting of the material. Similarly to the mixed boundary conditions, the inequality constraints in $(\mathrm{P})$ are essential for a realistic model, as demonstrated by the numerical example within this paper. Problem $(\mathrm{P})$ underlies various applications, such as the heat treatment of steel by means of an electric current. The example considered in the numerical part of this paper will deal with an application of this type.

The state system (1.1)-(1.7) exhibits some nonstandard features, particularly due to the quasi-linear coupling of the parabolic and the elliptic PDEs, the mixed boundary conditions in (1.5)-(1.7), and the inhomogeneity in the heat equation (1.1). A slightly different version of the thermistor problem is discussed by Chipot and collaborators (see [3] and the references therein). The system considered in [3] differs from (1.1)-(1.7), since it accounts for temperature dependent heat conductivities but does not allow for mixed boundary conditions and nonsmooth domains. The discussion of the state system (1.1)-(1.7) heavily rests on maximum elliptic and parabolic regularity results as derived in Gröger [17, 18]. Based on these results, it is possible to prove continuity of the temperature as a solution of (1.1)-(1.7), which is essential in the presence of pointwise state constraints as the inequality constraints on $\theta$ in (P). In particular, the application of Gröger's results implies the restriction to twodimensional domains, since comparable results for the three-dimensional case are not available.

To the best of the authors' knowledge, there are only a few contributions dealing with the optimal control of the thermistor problem. We refer to [23, 10, 22], where, similarly to our setting, two-dimensional problems are discussed. In [23], a complete parabolic problem is discussed, while [22] considers the purely elliptic counterpart to (1.1)-(1.7). In [10] and [28], the authors investigate a parabolic-elliptic system similar to (1.1)-(1.7), assuming a particular structure of the controls. In contrast to [23] and [22], mixed boundary conditions are considered in [10]. However, none of these works considers pointwise state constraints and nonsmooth data. Thus, (P) differs significantly from the above-mentioned papers. 
Problem (P) represents a quasi-linearly coupled state-constrained optimal control problem. Such optimization problems are known to provide particular difficulties, especially due to the pointwise state constraints. In the semilinear case, the analysis of state-constrained optimal control problems is already quite comprehensive; we mention only $[4,7,27,6]$ and the references therein. Concerning the state-constrained optimal control of semilinear elliptic PDEs with mixed boundary conditions, we refer to the recent publication [20]. In contrast to the semilinear case, less is known for the control of quasi-linear PDEs. Concerning quasi-linear, elliptic problems with pointwise state constraints, we refer to [8]. Hence, the discussion of optimal control of a quasi-linearly coupled PDE system in the presence of pointwise state-constraints and mixed boundary conditions represents a genuine contribution to the theory. Here we focus on the first-order analysis of $(\mathrm{P})$. The derivation of second-order sufficient conditions in the case of state-constrained boundary control of instationary problems is still an open question, even in the semilinear case with smooth data.

The paper is organized as follows. After stating the detailed setting and assumptions in section 2, the state system is discussed in section 3. The existence of an optimal solution is shown in section 4 , while section 5 is devoted to the analysis of the linearized state system. In section 6 , the first-order analysis of $(\mathrm{P})$ is developed, beginning with the differentiability of the control-to-state operator in section 6.1, followed by the discussion of the adjoint system and the derivation of the optimality system in sections 6.2 and 6.3, respectively. Finally, some numerical examples for a particular application problem covered by $(\mathrm{P})$ are presented in section 7 .

2. Notation and general assumptions. In all of what follows, $\Omega$ always denotes a domain in $\mathbb{R}^{2}$, and $\Gamma_{D}$ is a closed part of its boundary. The space $C^{\delta}(\Omega)$ denotes the space of Hölder continuous functions, while $H^{s, q}(\Omega)(s \in[0,1])$ is the space of Bessel potentials with differential index $s$ and summability index $q$ on the set $\Omega$. (Please notice that $H^{1, q}(\Omega)$ coincides with the Sobolev space $W^{1, q}(\Omega)$.) Further, we use the symbol $H_{D}^{s, q}(\Omega)$ for the closure of $\left\{\left.\psi\right|_{\Omega}: \psi \in C_{0}^{\infty}\left(\mathbb{R}^{2}\right)\right.$, supp $\left.\psi \cap \Gamma_{D}=\emptyset\right\}$ in $H^{s, q}(\Omega)$. The dual of $H_{D}^{1, q}(\Omega)$ with respect to the $L^{2}(\Omega)$ inner product is denoted by $H_{D}^{-1, q^{\prime}}(\Omega)$ with $\frac{1}{q}+\frac{1}{q^{\prime}}=1$. The dual of $H^{1, q^{\prime}}(\Omega)$ is denoted by $H_{\Omega}^{-1, q}(\Omega)$. If $\Omega$ is understood, we abbreviate $H^{s, q}, H_{D}^{s, q}$, and $H_{\Omega}^{-1, q}$, respectively. The symbol $S \subset \mathbb{R}$ always stands for an (open) interval. If $X$ is a Banach space, its dual is denoted by $X^{*}$. Moreover, $W^{1, r}(S ; X)$ is the set of those elements from $L^{r}(S ; X)$ whose distributional derivative also belongs to $L^{r}(S ; X)$. In this spirit, $\frac{\partial}{\partial t}$ always means the distributional derivative with respect to time; see [1, Chap. III.1] or [13, Chap. IV]. Furthermore, $C^{\tau}(S ; X)$ denotes the space of $X$-valued, Hölder continuous functions on $S$. For all these spaces, defined on an interval $S=] 0, T\left[\right.$, the subscript ${ }_{0}$ denotes the corresponding subspace of functions which vanish in $t=0$. All function spaces under our consideration are real. For two Banach spaces $X$ and $Y$ we denote the space of linear bounded operators from $X$ into $Y$ by $\mathcal{B}(X ; Y)$. The norm in a Banach space $X$ will be always indicated by $\|\cdot\|_{X}$. If $X, Y$ are Banach spaces which form an interpolation couple, then we denote by $[X, Y]_{\tau}$ the corresponding complex interpolation space and by $(X, Y)_{\tau, r}$ the real interpolation space; see [31]. Finally $c$ denotes a generic positive constant.

Now we are in position to state the main assumptions for the quantities in $(\mathrm{P})$. Please notice that, in order to obtain sharp results, here we mention just the assumptions on the quantities in (1.1)-(1.7) that are needed to obtain existence, uniqueness, and continuity of solutions to the state system. For the Fréchet-differentiability of 
the associated solution operator one has to require more restrictive conditions, which are formulated in Assumption 5.1; see section 5. We start with the conditions on the domain $\Omega$, as follows.

Assumption 2.1. The domain $\Omega \subset \mathbb{R}^{2}$ is a bounded Lipschitz domain (see [16]), and $\Gamma_{N}$ is an open part of $\partial \Omega$, whereas $\Gamma_{D}$ is a closed part of $\partial \Omega$. Furthermore $\Gamma_{N}$ and $\Gamma_{D}$ have positive measure and are disjoint to each other. In addition, the set $\Omega \cup\left(\partial \Omega \backslash \Gamma_{D}\right)$ is regular in the sense of Gröger (cf. [17]), which means in detail that for every point $x \in \partial \Omega$ there is an open neighborhood $\mathcal{U}_{x} \subset \mathbb{R}^{2}$ of $x$ and a bi-Lipschitz mapping $\Psi_{x}: \mathcal{U}_{x} \rightarrow \mathbb{R}^{2}$ such that $\Psi_{x}(x)=0 \in \mathbb{R}^{2}$ and $\Psi_{x}\left(\mathcal{U}_{x} \cap\left(\Omega \cup\left(\partial \Omega \backslash \Gamma_{D}\right)\right)\right)$ equals either the open half cube $\mathcal{C}:=\left\{y \in \mathbb{R}^{2}:-1<y_{1}<1,-1<y_{2}<0\right\}$ or $\mathcal{C} \cup \Upsilon$ or $\mathcal{C} \cup \Upsilon_{0}$, where $\Upsilon$ is the upper plate of $\mathcal{C}$ and $\Upsilon_{0}$ is the left half of $\Upsilon$.

Remark 2.2. In [20] it is shown that for $\Omega \cup\left(\partial \Omega \backslash \Gamma_{D}\right) \subset \mathbb{R}^{2}$ to be regular in the sense of Gröger it is necessary and sufficient that $\Omega$ be a Lipschitz domain and $\Gamma_{D}$ be a finite union of closed arc pieces from $\partial \Omega$, where none of these degenerates to a single point.

Assumption 2.3. On the quantities in the state system we impose the following:

(i) The function $\sigma(x, \theta): \Omega \times \mathbb{R} \rightarrow \mathcal{B}\left(\mathbb{R}^{2}\right)$ is bounded and measurable w.r.t. $x$ for all $\theta \in \mathbb{R}$ and Lipschitz continuous w.r.t. $\theta$ for almost all $x \in \Omega$; i.e.,

$$
\|\sigma(x, \tilde{\theta})-\sigma(x, \theta)\|_{\mathcal{B}\left(\mathbb{R}^{2}\right)} \leq L_{\sigma}|\tilde{\theta}-\theta| \quad \text { a.e. in } \Omega, \forall \tilde{\theta}, \theta \in \mathbb{R}
$$

with a constant $L_{\sigma}>0$. Moreover, for all $\theta \in \mathbb{R}$ and almost all $x \in \Omega, \sigma(x, \theta)$ is a symmetric matrix. Finally, $\sigma(x, \theta)$ satisfies

$$
\begin{aligned}
& \inf _{\theta \in \mathbb{R}} \operatorname{essinf}_{x \in \Omega} \sum_{i, j=1}^{2} \sigma_{i j}(x, \theta) \xi_{i} \xi_{j} \geq \sigma_{0}\|\xi\|_{\mathbb{R}^{2}} \quad \forall \xi \in \mathbb{R}^{2}, \\
& \sup _{\theta \in \mathbb{R}}\|\sigma(x, \theta)\|_{L^{\infty}\left(\Omega ; \mathcal{B}\left(\mathbb{R}^{2}\right)\right)} \leq \sigma_{1},
\end{aligned}
$$

with constants $0<\sigma_{0} \leq \sigma_{1}<\infty$.

(ii) The function $\kappa \in L^{\infty}\left(\Omega ; \mathcal{B}\left(\mathbb{R}^{2}\right)\right)$ is symmetric for almost all $x \in \Omega$ and satisfies the usual ellipticity condition; i.e.,

$$
\underset{x \in \Omega}{\operatorname{essinf}} \sum_{i, j=1}^{2} \kappa_{i j}(x) \xi_{i} \xi_{j} \geq \sigma_{0}\|\xi\|_{\mathbb{R}^{2}} \quad \forall \xi \in \mathbb{R}^{2} .
$$

(iii) $\theta_{l} \in L^{\infty}(] 0, T\left[; L^{\infty}(\partial \Omega)\right)$.

(iv) $\alpha \in L^{2}(\partial \Omega)$ with $\int_{\partial \Omega} \alpha^{2} d \omega>0$ and $\alpha(x) \geq 0$ a.e. on $\partial \Omega$.

(v) $\theta_{0} \in C(\bar{\Omega})$.

Assumption 2.4. The remaining quantities in $(\mathrm{P})$ fulfill the following:

(i) $D$ is an open (not necessarily proper) subset of $\Omega$.

(ii) $\theta_{d} \in L^{2}(D)$.

(iii) $\theta_{\max } \in C(\bar{Q})$ with $\theta_{0}(x)<\theta_{\max }(x, 0)$ for all $x \in \bar{\Omega}$.

(iv) $u_{\max } \in L^{\infty}(] 0, T\left[; L^{2}\left(\Gamma_{N}\right)\right), u_{\max }(x, t) \geq 0$ a.e. on $\Sigma_{N}$.

(v) $\beta>0$.

Remark 2.5. We point out that the conditions in Assumptions 2.1, 2.3, and 2.4 are satisfied in many relevant cases. In particular, allowing for nonsmooth domains is important in many applications. 
3. Analysis of the nonlinear state system. We start with a precise formulation of system (1.1)-(1.7) and the corresponding definition of weak solutions to (1.1)-(1.7). To this end, define for any coefficient function $\rho \in L^{\infty}\left(\Omega ; \mathcal{B}\left(\mathbb{R}^{2}\right)\right)$

$$
-\nabla \cdot \rho \nabla: H_{D}^{1,2}(\Omega) \rightarrow H_{D}^{-1,2}(\Omega)
$$

by

$$
\langle-\nabla \cdot \rho \nabla w, z\rangle:=\int_{\Omega} \rho \nabla w \cdot \nabla z d x, \quad w, z \in H_{D}^{1,2}(\Omega)
$$

The restriction of these operators to the spaces $H_{D}^{1, q}(q \geq 2)$ will also be denoted by $-\nabla \cdot \rho \nabla$. Analogously, we define

$$
K: H^{1,2}(\Omega) \rightarrow H_{\Omega}^{-1,2}(\Omega)
$$

by

$$
\langle K w, z\rangle:=\int_{\Omega} \kappa \nabla w \cdot \nabla z d x+\int_{\partial \Omega} \alpha w z d w, \quad w, z \in H^{1,2}(\Omega),
$$

where (here and in what follows) $\omega$ is the surface measure on $\partial \Omega$.

Remark 3.1. The function

$$
\iota_{0}:\left[0, \infty\left[\ni t \mapsto e^{-t K} \theta_{0} \in L^{\infty}(\Omega)\right.\right.
$$

is continuous on $] 0, \infty\left[\right.$ and admits the estimate $\left\|e^{-t K} \theta_{0}\right\|_{L^{\infty}} \leq\left\|\theta_{0}\right\|_{L^{\infty}}$; see Lemma 3.20 below.

Remark 3.2. For $q \in\left[2,4\left[\right.\right.$ one has the embedding $H^{1, q^{\prime}}(\Omega) \hookrightarrow L^{2+\varepsilon}(\partial \Omega)$ for an $\varepsilon=\varepsilon(q)>0$ and $H^{1,2}(\Omega) \hookrightarrow L^{m}(\partial \Omega)$ for any finite $m$; see [16]. Assume now $\varrho \in L^{2}(\partial \Omega)$. If one sets $m:=\frac{2(2+\varepsilon)}{\varepsilon}$, then there is a constant $c>0$ such that, for all $v \in H^{1,2}(\Omega)$ and all $\psi \in H^{1, q^{\prime}}(\Omega)$,

$$
\begin{aligned}
\left|\int_{\partial \Omega} \varrho v \psi d \omega\right| & \leq\|\varrho\|_{L^{2}(\partial \Omega)}\|v\|_{L^{m}(\partial \Omega)}\|\psi\|_{L^{2+\epsilon}(\partial \Omega)} \\
& \leq c\|\varrho\|_{L^{2}(\partial \Omega)}\|v\|_{H^{1,2}(\Omega)}\|\psi\|_{H^{1, q^{\prime}}(\Omega)} .
\end{aligned}
$$

This estimate gives the following two results:

(a) Setting $v \equiv 1$, one can associate with the $L^{2}(\partial \Omega)$ function $\alpha \theta_{l}(t)$ for almost all $t \in] 0, \infty\left[\right.$ an element from $H_{\Omega}^{-1, q}(\Omega)$, provided $q \in[2,4[$. In this spirit we will understand $\alpha \theta_{l} \in L^{\infty}(] 0, T\left[; L^{2}(\partial \Omega)\right)$ as an element $\tilde{\alpha} \in L^{\infty}(] 0, T\left[; H_{\Omega}^{-1, q}(\Omega)\right)$. In the same way a function $u \in L^{\infty}(] 0, T\left[; L^{2}\left(\Gamma_{N}\right)\right)$ will be interpreted as an element $\tilde{u} \in L^{\infty}(] 0, T\left[; H_{D}^{-1, q}\right)$ by setting

$$
\langle\tilde{u}(t), v\rangle_{H_{D}^{-1, q}, H_{D}^{1, q^{\prime}}}=\int_{\Gamma_{N}} u(t) v d s, \quad v \in H_{D}^{1, q^{\prime}}(\Omega),
$$

for almost all $t \in] 0, \infty[$.

(b) The estimate (3.6) shows that $\varrho \in L^{2}(\partial \Omega)$ induces a (continuous) linear mapping $\Phi_{\varrho}: H^{1,2}(\Omega) \rightarrow H_{\Omega}^{-1, q}$ given by $\left\langle\Phi_{\varrho} v, \psi\right\rangle:=\int_{\partial \Omega} \varrho v \psi d \omega$.

Now we are in a position to give the precise definition of solutions to (1.1)-(1.7) in a weak sense.

Copyright $@$ by SIAM. Unauthorized reproduction of this article is prohibited. 
Definition 3.3. Let $u$ be a given function in $L^{\infty}(] 0, T\left[; L^{2}\left(\Gamma_{N}\right)\right)$. We consider $(\theta, \varphi)$ as a solution of (1.1)-(1.7) if there are indices $q \in] 2,4[$ and $r \in] 1, \infty[$ such that

$$
\begin{gathered}
\varphi \in L^{\infty}(] 0, T\left[; H_{D}^{1, q}\right), \\
\zeta:=\theta-\iota_{0} \in W_{0}^{1, r}(] 0, T\left[; H_{\Omega}^{-1, q}\right) \cap L^{r}(] 0, T\left[; H^{1, q}\right),
\end{gathered}
$$

and, additionally, the operator equations

$$
\begin{gathered}
\frac{\partial \zeta}{\partial t}+K \zeta=\left(\sigma\left(\zeta+\iota_{0}\right) \nabla \varphi\right) \cdot \nabla \varphi+\tilde{\alpha}, \\
-\nabla \cdot \sigma\left(\zeta+\iota_{0}\right) \nabla \varphi=\tilde{u}
\end{gathered}
$$

are satisfied.

Remark 3.4. The reader will verify that the boundary conditions imposed on $\theta$ and $\varphi$ in (1.2), (1.5), (1.7) are incorporated in this definition in the spirit of [13, Chap. II.2] or [9, Chap. 1.2], for instance.

The main result, which we will show in this section, reads as follows.

TheOrem 3.5. Suppose that $u$ is given in $L^{\infty}(] 0, T\left[; L^{2}\left(\Gamma_{N}\right)\right)$. Then under Assumptions 2.1, 2.3, and 2.4 the following hold:

(i) There is a solution of (1.1)-(1.7) in the sense of Definition 3.3.

(ii) This solution is unique.

(iii) There is an index $\eta>0$ such that for every $T>0$ the function $\zeta$ even belongs to $C^{\eta}(] 0, T\left[; C^{\eta}(\Omega)\right)$.

(iv) If $\theta_{0} \in H^{\varsigma, q}(\Omega)$ with $\varsigma>\frac{2}{q}$, then $\iota_{0}$ takes its values in a Hölder space $C^{\eta}(\Omega)$ and is Hölderian in time when considered as $C^{\eta}(\Omega)$-valued, which means $\zeta+$ $\iota_{0} \in C^{\eta}(] 0, T\left[; C^{\eta}(\Omega)\right)$ if $\eta>0$ is sufficiently small.

Remark 3.6. Please notice that the Hölder property of $\theta$ in case (iv) extends to the boundaries, i.e., $\theta \in C^{\eta}\left([0, T] ; C^{\eta}(\bar{\Omega})\right)$, and naturally implies continuity of $\theta$. This is essential for the derivation of first-order necessary conditions for $(\mathrm{P})$; see section 6 .

3.1. Proof of Theorem 3.5. Let us start with a brief sketch of the proof. In contrast to [3], where Schauder's fixed point theorem is used to analyze the thermistor problem, we here apply Banach's contraction principle to prove existence and uniqueness for (1.1)-(1.7). The associated fixed point mapping is constructed as follows: Let $\mathcal{J}: \theta \mapsto \varphi$ be the solution operator associated with the elliptic equation (3.10) for given $u$, while $\mathcal{K}: f \mapsto \theta$ is the solution operator of the parabolic equation (3.9) with right-hand side $f$. (The precise definitions of $\mathcal{J}$ and $\mathcal{K}$ with their domains and ranges, respectively, will be given later on; see Lemmas 3.9, 3.11, and 3.21.) Then a solution of (1.1)-(1.7) in the sense of Definition 3.3 is equivalent to a fixed point of the equation

$$
\theta=\mathcal{K}\left(\sigma(\tilde{\theta})|\nabla \mathcal{J}(\tilde{\theta})|^{2}+\tilde{\alpha}\right) .
$$

To prove contractivity of this combined mapping, we apply maximal elliptic and parabolic regularity results in the spirit of Gröger [17, 18]. These results in particular allow us to account for the mixed boundary conditions in the elliptic equation. The contractivity will first be shown on sufficiently small time intervals. A repetition argument then implies the assertion of Theorem 3.5 on the whole time interval.

The proof is organized as follows. We start with the discussion of the elliptic equation. Afterwards the parabolic equation is investigated, starting with a summary of some well-known results on semigroup theory and maximum parabolic regularity, which are proven in the appendix. Finally the fixed point mapping is constructed, and the contractivity is shown. Throughout the proof, let $T_{0}$ and $T_{1}$ be fixed but 
arbitrary numbers satisfying $0 \leq T_{0}<T_{1}<\infty$. The interval $] T_{0}, T_{1}[$ is denoted by $S$. Moreover, by $\iota_{T_{0}}$ we denote the mapping $\iota_{0}\left(\cdot-T_{0}\right)$.

For the discussion of the elliptic equation, we employ a result of Gröger [17]. It covers maximal regularity for elliptic equations.

THEOREM 3.7. Assume that $\Omega \cup\left(\partial \Omega \backslash \Gamma_{D}\right)$ is regular in the sense of Gröger (see Assumption 2.1 and Remark 2.2), and let $\underline{\rho}, \bar{\rho}$ be two positive constants, satisfying $\underline{\rho} \leq \bar{\rho}$.

(i) Then there is a $q_{0}>2$ such that for every $q \in\left[2, q_{0}\right]$ and for all coefficient functions $\rho$ with ellipticity constant not smaller than $\underline{\rho}$ and $\|\rho\|_{L^{\infty}\left(\Omega ; \mathcal{B}\left(\mathbb{R}^{2}\right)\right)} \leq \bar{\rho}$ the operator $-\nabla \cdot \rho \nabla$ provides a topological isomorphism between $H_{D}^{1, q}(\Omega)$ and $H_{D}^{-1, q}(\Omega)$.

(ii) For each $q \in\left[2, q_{0}\right]$ the numbers $\left\|(-\nabla \cdot \rho \nabla)^{-1}\right\|_{\mathcal{B}\left(H_{D}^{-1, q} ; H_{D}^{1, q}\right)}$ are uniformly bounded within the class of all such coefficient functions $\rho$.

Now, let $\tilde{q}_{0}$ be the number $q_{0}$ from Theorem 3.7 which corresponds to $\rho:=\sigma_{0}$ and $\bar{\rho}:=\sigma_{1}$, where $\sigma_{0}$ and $\sigma_{1}$ are the constants taken from Assumption 2.3, and fix a $q$ from $] 2, \min \left(\tilde{q}_{0}, 4\right)[$.

Remark 3.8. It is well known from the theory of mixed boundary value problems that a number $q_{0} \geq 4$ cannot be expected in the context of Theorem 3.7.

With Theorem 3.7 at hand, we can introduce the first part of our fixed point mapping, namely the solution operator associated with the elliptic equation.

LEMMA 3.9.

(i) The mapping $\mathcal{J}$ which assigns to any function $\zeta \in L^{\infty}\left(S ; L^{\infty}(\Omega)\right)$ the function $t \mapsto \varphi_{t}$ with $\varphi_{t}$ given by

$$
-\nabla \cdot \sigma\left(\zeta(t)+\iota_{T_{0}}(t)\right) \nabla \varphi_{t}=\tilde{u}(t)
$$

takes its image in a ball $B$ in $L^{\infty}\left(S ; H_{D}^{1, q}(\Omega)\right)$, the radius of which depends only on $\|u\|_{L^{\infty}\left(S ; L^{2}\left(\Gamma_{N}\right)\right)}$ (not on $\theta_{0} \in L^{\infty}(\Omega)$ and the interval $S$ ).

(ii) $\mathcal{J}: L^{\infty}\left(S ; L^{\infty}(\Omega)\right) \rightarrow B$ is Lipschitz continuous.

Proof. (i) First, Remark 3.1 shows that $\iota_{T_{0}}$ has globally in time the $L^{\infty}(\Omega)$ bound $\left\|\theta_{0}\right\|_{L^{\infty}(\Omega)}$. Moreover, for almost all $t \in S, u(t) \in L^{2}\left(\Gamma_{N}\right)$ defines an element $\tilde{u}(t) \in H_{D}^{-1, q}(\Omega)$; see Remark 3.2. Thus, the first assertion follows from Theorem 3.7 and the uniform boundedness of $\sigma$.

(ii) Let $\zeta, \tilde{\zeta} \in L^{\infty}\left(S ; L^{\infty}(\Omega)\right)$ be fixed but arbitrary. For almost all $t \in S$, we estimate

$$
\begin{aligned}
& \left\|\tilde{\varphi}_{t}-\varphi_{t}\right\|_{H_{D}^{1, q}} \\
& =\left\|\left[\left(\nabla \cdot \sigma\left(\tilde{\zeta}(t)+\iota_{T_{0}}(t)\right) \nabla\right)^{-1}-\left(\nabla \cdot \sigma\left(\zeta(t)+\iota_{T_{0}}(t)\right) \nabla\right)^{-1}\right] \tilde{u}(t)\right\|_{H_{D}^{1, q}} \\
& =\|\left(\nabla \cdot \sigma\left(\tilde{\zeta}(t)+\iota_{T_{0}}(t)\right) \nabla\right)^{-1}\left(\nabla \cdot\left(\sigma\left(\tilde{\zeta}(t)+\iota_{T_{0}}(t)\right)-\sigma\left(\zeta(t)+\iota_{T_{0}}(t)\right)\right) \nabla\right) \\
& \quad\left(\nabla \cdot \sigma\left(\zeta(t)+\iota_{T_{0}}(t)\right) \nabla\right)^{-1} \tilde{u}(t) \|_{H_{D}^{1, q}} \\
& \leq\left\|\left(\nabla \cdot \sigma\left(\tilde{\zeta}(t)+\iota_{T_{0}}(t)\right) \nabla\right)^{-1}\right\|_{\mathcal{B}\left(H_{D}^{-1, q} ; H_{D}^{1, q}\right)}\left\|\left(\nabla \cdot \sigma\left(\zeta(t)+\iota_{T_{0}}(t)\right) \nabla\right)^{-1}\right\|_{\mathcal{B}\left(H_{D}^{-1, q} ; H_{D}^{1, q}\right)} \\
& \left\|\left(\sigma\left(\tilde{\zeta}(t)+\iota_{T_{0}}(t)\right)-\sigma\left(\zeta(t)+\iota_{T_{0}}(t)\right)\right)\right\|_{L^{\infty}\left(\Omega ; \mathcal{B}\left(\mathbb{R}^{2}\right)\right)}\|\tilde{u}(t)\|_{H_{D}^{-1, q}} \\
& \leq\left\|\left(\nabla \cdot \sigma\left(\tilde{\zeta}(t)+\iota_{T_{0}}(t)\right) \nabla\right)^{-1}\right\|_{\mathcal{B}\left(H_{D}^{-1, q} ; H_{D}^{1, q}\right)}\left\|\left(\nabla \cdot \sigma\left(\zeta(t)+\iota_{T_{0}}(t)\right) \nabla\right)^{-1}\right\|_{\mathcal{B}\left(H_{D}^{-1, q} ; H_{D}^{1, q}\right)} \\
& L_{\sigma}\|\tilde{\zeta}-\zeta\|_{L^{\infty}\left(S ; L^{\infty}(\Omega)\right)}\|\tilde{u}(t)\|_{H_{D}^{-1, q},}
\end{aligned}
$$

where $L_{\sigma}$ denotes the Lipschitz constant of $\sigma$. Thus the proof is complete. 
Remark 3.10. Please notice that none of these estimates depends on the interval $S$ nor on the initial value $\theta_{0} \in L^{\infty}(\Omega)$.

The next lemma incorporates the right-hand side of the parabolic equation (3.9) into the fixed point mapping.

Lemma 3.11. The mapping

$$
\mathcal{G}: L^{\infty}\left(S ; L^{\infty}(\Omega)\right) \ni \zeta \mapsto \sigma\left(\zeta+\iota_{T_{0}}\right) \nabla \mathcal{J}(\zeta) \cdot \nabla \mathcal{J}(\zeta) \in L^{\infty}\left(S ; L^{q / 2}(\Omega)\right)
$$

is Lipschitzian, and its image is contained in a ball $M \subset L^{\infty}\left(S ; L^{q / 2}(\Omega)\right)$.

Proof. Suppose that $\varphi, \tilde{\varphi} \in H_{D}^{1, q}(\Omega)$ and $\theta \in \mathbb{R}$ are fixed but arbitrary. Using Minkowski's and Hölder's inequalities, we find

$$
\begin{aligned}
& \left(\int_{\Omega}|(\sigma(\theta) \nabla \varphi) \cdot \nabla \varphi-(\sigma(\theta) \nabla \tilde{\varphi}) \cdot \nabla \tilde{\varphi}|^{q / 2}\right)^{2 / q} \\
& \leq\left(\int_{\Omega}(|(\sigma(\theta) \nabla \varphi) \cdot \nabla(\varphi-\tilde{\varphi})|+|(\sigma(\theta) \nabla(\varphi-\tilde{\varphi})) \cdot \nabla \tilde{\varphi}|)^{q / 2}\right)^{2 / q} \\
& \leq \sigma_{1}\left[\left(\int_{\Omega}|\nabla \tilde{\varphi}|^{q} d x\right)^{1 / q}+\left(\int_{\Omega}|\nabla \varphi|^{q} d x\right)^{1 / q}\right]\left(\int_{\Omega} \mid \nabla\left(\varphi-\left.\tilde{\varphi}\right|^{q}\right)^{1 / q} .\right.
\end{aligned}
$$

Now let $\zeta, \tilde{\zeta} \in L^{\infty}\left(S ; L^{\infty}(\Omega)\right)$ be fixed but arbitrary. Hence $\mathcal{J}(\zeta), \mathcal{J}(\tilde{\zeta}) \in B \subset$ $L^{\infty}\left(S ; H_{D}^{1, q}\right)$. In view of (3.13), we then find

$$
\begin{gathered}
\left\|\left(\sigma\left(\zeta+\iota_{T_{0}}\right) \nabla \mathcal{J}(\zeta)\right) \cdot \nabla \mathcal{J}(\zeta)-\left(\sigma\left(\tilde{\zeta}+\iota_{T_{0}}\right) \nabla \mathcal{J}(\tilde{\zeta})\right) \cdot \nabla \mathcal{J}(\tilde{\zeta})\right\|_{L^{\infty}\left(S ; L^{q / 2}\right)} \\
\leq\left\|\left(\sigma\left(\zeta+\iota_{T_{0}}\right) \nabla \mathcal{J}(\zeta)\right) \cdot \nabla \mathcal{J}(\zeta)-\left(\sigma\left(\zeta+\iota_{T_{0}}\right) \nabla \mathcal{J}(\tilde{\zeta})\right) \cdot \nabla \mathcal{J}(\tilde{\zeta})\right\|_{L^{\infty}\left(S ; L^{q / 2}\right)} \\
\left\|\left[\sigma\left(\zeta+\iota_{T_{0}}\right)-\sigma\left(\tilde{\zeta}+\iota_{T_{0}}\right)\right] \nabla \mathcal{J}(\tilde{\zeta}) \cdot \nabla \mathcal{J}(\tilde{\zeta})\right\|_{L^{\infty}\left(S ; L^{q / 2}\right)} \\
\leq \sigma_{1}\left(\|\mathcal{J}(\zeta)\|_{L^{\infty}\left(S ; H_{D}^{1, q}\right)}+\|\mathcal{J}(\tilde{\zeta})\|_{L^{\infty}\left(S ; H_{D}^{1, q}\right)}\right)\|\mathcal{J}(\zeta)-\mathcal{J}(\tilde{\zeta})\|_{L^{\infty}\left(S ; H_{D}^{1, q}\right)} \\
\quad+L_{\sigma}\|\zeta-\tilde{\zeta}\|_{L^{\infty}\left(S ; L^{\infty}(\Omega)\right)}\|\nabla \mathcal{J}(\tilde{\zeta}) \cdot \nabla \mathcal{J}(\tilde{\zeta})\|_{L^{\infty}\left(S ; L^{q / 2}\right)} .
\end{gathered}
$$

Thus, Lemma 3.9 gives the first assertion.

Remark 3.12. An inspection of the above arguments shows that neither the radius of $M$ nor the Lipschitz constant depends on the initial value $\theta_{0} \in L^{\infty}(\Omega)$ or the interval $S$.

For the definition of the solution operator associated with the parabolic equation, which is the last part of our fixed point mapping (cf. (3.11)), some essential results on semigroup theory and maximal parabolic regularity are required. For the convenience of the reader, we collect these in what follows. The associated proofs are postponed to the appendix.

Lemma 3.13. Let $A$ be a generator of an analytic semigroup on a Banach space $X$ and $0 \notin \operatorname{spec}(A)$ (so that the graph norm on $\mathcal{D}$ induced by $A$ is equivalent to the norm $\left.\|A \cdot\|_{X}\right)$. Then the following hold:

(i) For every $x \in X$ and every $\left.T_{0}, T_{1} \in\right] 0, \infty[$ the function

$$
] T_{0}, T_{1}\left[\ni t \mapsto e^{-t A} x \in \mathcal{D}\right.
$$

is Lipschitzian.

(ii) If $x \in[X, \mathcal{D}]_{\tau}$ and $\left.\rho \in\right] 0, \tau[$, then the function

$$
] 0, T\left[\ni t \mapsto e^{-t A} x \in[X, \mathcal{D}]_{\rho}\right.
$$

is from $C^{\tau-\rho}(] 0, T\left[;[X, \mathcal{D}]_{\rho}\right)$ for any finite $T>0$.

Copyright $@$ by SIAM. Unauthorized reproduction of this article is prohibited. 
The proof of Lemma 3.13 is fairly standard and given in the appendix. Let us next recall the concept of maximal regularity and point out some basic facts, as follows.

Definition 3.14. Let $X$ be a Banach space and $A$ be a closed operator with dense domain $\mathcal{D} \subset X$ and $S=] T_{0}, T_{1}[\subset \mathbb{R}$ a bounded interval. Suppose $r \in] 1, \infty[$. Then we say that $A$ satisfies maximal parabolic $L^{r}(S ; X)$-regularity if for any $f \in L^{r}(S ; X)$ there is a unique function $w \in W_{0}^{1, r}(S ; X) \cap L^{r}(S ; \mathcal{D})$ which satisfies

$$
\frac{d w}{d t}+A w=f
$$

Remark 3.15. The following results on maximal parabolic $L^{r}(S ; X)$-regularity are known:

(a) If $A$ satisfies maximal parabolic $L^{r}(S ; X)$-regularity, then it does so for any other (bounded) interval (see [12]).

(b) If $A$ satisfies maximal parabolic $L^{r}(S ; X)$-regularity, then it satisfies maximal parabolic $L^{s}(S ; X)$-regularity for all $\left.s \in\right] 1, \infty[$; see [30] or [12].

(c) There is a continuous injection

$$
\mathcal{E}: W^{1, r}(S ; X) \cap L^{r}(S ; \mathcal{D}) \hookrightarrow C\left(\bar{S} ;(X, \mathcal{D})_{1-\frac{1}{r}, r}\right) ;
$$

see [1, Chap. III, Thm. 4.10.2] and [31, Chap. 1.8].

LEMMA 3.16.

(i) Assume that $A$ satisfies maximal parabolic $L^{r}(S ; X)$-regularity. Let $\mathcal{L}$ be the operator which assigns to any right-hand side $f \in L^{r}(S ; X)$ the solution $w \in$ $W_{0}^{1, r}(S ; X) \cap L^{r}(S ; \mathcal{D})$ of $(3.16)$. Then the norm of $\mathcal{L}$ does not increase when the interval length shrinks.

(ii) Let $\mathcal{E}_{0}$ denote the restriction of $\mathcal{E}$ to the subspace $\left\{\psi: \psi\left(T_{0}\right)=0\right\}$; then the norm of $\mathcal{E}_{0}$ does not increase if the interval length shrinks.

As for the proof of Lemma 3.13, the corresponding proof is postponed to the appendix. We continue with the following lemma. Its proof is based on the theory of interpolation spaces and also given in the appendix.

LEMMA 3.17.

(i) For any $\eta \in] 0,1-\frac{1}{r}[$ there is a continuous embedding

$$
(X, \mathcal{D})_{1-\frac{1}{r}, r} \hookrightarrow[X, \mathcal{D}]_{\eta}
$$

and, consequently, a continuous embedding

$$
\mathcal{E}_{C}: C\left(S ;(X, \mathcal{D})_{1-\frac{1}{r}, r}\right) \hookrightarrow C\left(S ;[X, \mathcal{D}]_{\eta}\right) .
$$

(ii) The norm of $\mathcal{E}_{C} \mathcal{E}_{0} \mathcal{L}$ does not increase if the interval length shrinks.

(iii) Assume $\tau \in] 0,1-\frac{1}{r}\left[\right.$. Then there is an index @ such that $W^{1, r}(S ; X) \cap$ $L^{r}(S ; \mathcal{D})$ even continuously embeds into $C^{\varrho}\left(S ;[X, \mathcal{D}]_{\tau}\right)$.

In order to apply the concept of maximal parabolic regularity to our situation, we need the following result.

THEOREM 3.18. There is a $\left.q_{1} \in\right] 2,4\left[\right.$ such that for every $q \in\left[2, q_{1}\right]$ and every $S \subset] 0, T$ [ the operator $K$, defined in (3.4), satisfies maximal parabolic $L^{r}\left(S ; H_{\Omega}^{-1, q}\right)$ regularity with $H^{1, q}$ being the domain $\mathcal{D}$ of $K$.

Proof. The theorem is proved in [18] for the case $r=q$; namely, it is first shown if only $A=-\nabla \cdot \kappa \nabla$ and afterwards extended to perturbed operators $A+F$, provided that $F$ is a mapping from $H^{1,2}(\Omega)$ into $H_{\Omega}^{-1, q}(\Omega)$; see Remark 5 of [18]. That this 
is indeed the case for the $\alpha$-term in (3.4) was shown in Remark 3.2(b). The case of arbitrary $r \in] 1, \infty[$ is obtained by Remark 3.15(ii).

Remark 3.19. In all of what follows, let $q$ be a fixed number in $] 2, \min \left(\bar{q}_{0}, q_{1}, 4\right)[$, where $\bar{q}_{0}$ is the number $q_{0}$ from Theorem 3.7 associated with $\sigma_{0}$ and $\sigma_{1}$.

The next result concerns an a priori estimate for the function $\iota_{0}$, as defined in Remark 3.1. The associated proof is based on the theory of semigroups and presented in the appendix.

LEMma 3.20. Let us (as above) denote the function $\left[0, \infty\left[\ni t \mapsto e^{-t K} \theta_{0}\right.\right.$ by $\iota_{0}$, where $K$ is as defined in (3.4). Moreover, let $q>0$ be the number defined in the above remark.

(i) If $\theta_{0} \in L^{\infty}$, then $\iota_{0}$ admits the estimate $\left\|e^{-t K} \theta_{0}\right\|_{L^{\infty}} \leq\left\|\theta_{0}\right\|_{L^{\infty}}$. For $t>0$, $\iota_{0}(t)$ even belongs to $H^{1, q}$ and the restriction of $\iota_{0}$ to any interval $] T_{0}, T_{1}[$ with $0<T_{0}<T_{1}<\infty$ is Lipschitz continuous if $\iota_{0}$ is considered as $H^{1, q}$-valued.

(ii) If $\theta_{0} \in H^{\varsigma, q}(\Omega)$ with $\varsigma>\frac{2}{q}$, then $\iota_{0}$ takes its values in a Hölder space $C^{\eta}(\Omega)$ and is Hölderian in time when considered as $C^{\eta}(\Omega)$-valued.

Following the notation of Lemma 3.16, we denote by $\mathcal{L}$ the operator that assigns to a given right-hand side $f \in L^{r}\left(S ; H_{\Omega}^{-1, q}\right)$ the solution $\zeta \in W_{0}^{1, r}\left(S ; H_{\Omega}^{-1, q}\right) \cap L^{r}\left(S ; H^{1, q}\right)$ of

$$
\frac{\partial \zeta}{\partial t}+K \zeta=f
$$

Since $K$ satisfies maximal parabolic $L^{r}\left(S ; H_{\Omega}^{-1, q}\right)$-regularity, $\mathcal{L}$ is well defined, and the assertions of Lemmas 3.16 and 3.17 hold with $X=H_{\Omega}^{-1, q}$ and $\mathcal{D}=H^{1, q}$.

LEMMA 3.21. Let $\mathcal{F}$ denote the mapping

$$
L^{\infty}\left(S, L^{q / 2}\right) \ni f \mapsto f+\tilde{\alpha} \in L^{r}\left(S, H_{\Omega}^{-1, q}\right)
$$

(via the embedding $L^{q / 2} \hookrightarrow H_{\Omega}^{-1, q}$ ), and define $\mathcal{K}:=\mathcal{E}_{C} \mathcal{E}_{0} \mathcal{L} \mathcal{F}$. Then $\mathcal{K}$ is Lipschitzian, and its Lipschitz constant tends to zero as $\left(T_{1}-T_{0}\right) \rightarrow 0$, i.e., with shrinking time interval length.

Proof. Let $f, \tilde{f} \in L^{\infty}\left(S, L^{q / 2}\right)$ be given. The maximal parabolic regularity of $K$ then implies

$$
\begin{aligned}
\|\mathcal{K}(f)-\mathcal{K}(\tilde{f})\|_{C\left(S ;[X, \mathcal{D}]_{\eta}\right)} & \leq\left\|\mathcal{E}_{C} \mathcal{E}_{0} \mathcal{L}\right\|_{\mathcal{B}\left(L^{r}(S ; X) ; C\left(S ;[X, \mathcal{D}]_{\eta}\right)\right)}\|\mathcal{F}(f)-\mathcal{F}(\tilde{f})\|_{L^{r}\left(S ; H_{\Omega}^{-1, q}\right)} \\
& \leq c\left|T_{1}-T_{0}\right|^{1 / r}\|f-\tilde{f}\|_{L^{\infty}\left(S ; L^{q / 2}\right)}
\end{aligned}
$$

with a constant $c$ independent of $|S|=\left|T_{1}-T_{0}\right|$ because of Lemma 3.17.

With the above results we can now prove the contractivity of the fixed point mapping, as indicated at the beginning of this section. For this purpose, we consider the combined mapping

$$
\mathcal{K G}: L^{\infty}(] T_{0}, T_{1}\left[; L^{\infty}(\Omega)\right) \rightarrow L^{\infty}(] T_{0}, T_{1}\left[; L^{\infty}(\Omega)\right)
$$

and show that it is strictly contractive if $T_{1}-T_{0}$ is sufficiently small. Here $\mathcal{G}$ is the operator, defined in Lemma 3.11. In order to prove contractivity, let us define the number $r$ as $r>\frac{2 q}{q-2}$. Please notice that the interval $] \frac{1}{2}+\frac{1}{q}, 1-\frac{1}{r}[$ is then not empty due to $q>2$. Assume now $\eta \in] \frac{1}{2}+\frac{1}{q}, 1-\frac{1}{r}\left[\right.$; then $2 \eta-1>\frac{2}{q}$, and hence,

$$
\left[H^{-1, q}, H^{1, q}\right]_{\eta}=H^{2 \eta-1, q} \hookrightarrow C^{\varsigma}(\Omega) \hookrightarrow L^{\infty}(\Omega)
$$


with $\varsigma:=2 \eta-1-\frac{2}{q}>0$; see [31, Chap. 4.6.1]. Due to Lemmas 3.11 and 3.21 and (3.19), $\mathcal{K G}$ is well defined, and for all $\zeta, \tilde{\zeta} \in L^{\infty}\left(S ; L^{\infty}(\Omega)\right)$ there holds

$$
\|\mathcal{K G}(\zeta)-\mathcal{K G}(\tilde{\zeta})\|_{L^{\infty}\left(S ; L^{\infty}(\Omega)\right)} \leq c\left|T_{1}-T_{0}\right|^{1 / r}\|\zeta-\tilde{\zeta}\|_{L^{\infty}\left(S ; L^{\infty}(\Omega)\right)} .
$$

Thus $\mathcal{K G}$ is contractive if $T_{1}-T_{0}<\varepsilon$, provided that $\varepsilon$ is sufficiently small. Therefore, the fixed point equation $\zeta=\mathcal{K} \mathcal{G}(\zeta)$ must have a unique solution by Banach's contraction principle if $\varepsilon$ is small enough. Please notice that $\varepsilon$ depends neither on $T_{0}$ nor on $\theta_{0}$. Moreover, by construction, this fixed point equation is equivalent to the following system of operator equations on $] T_{0}, T_{0}+\varepsilon[$ :

$$
\begin{aligned}
\frac{\partial \zeta}{\partial t}+K \zeta & =\left(\sigma\left(\zeta+\iota_{T_{0}}\right) \nabla \varphi\right) \cdot \nabla \varphi+\tilde{\alpha} \quad \text { in } H_{\Omega}^{-1, q}, \\
-\nabla \cdot \sigma\left(\zeta+\iota_{T_{0}}\right) \nabla \varphi & =\tilde{u} \quad \text { in } H_{D}^{-1, q} .
\end{aligned}
$$

Hence, the fixed point is identical with the unique solution

$$
\begin{gathered}
\varphi \in L^{\infty}(] T_{0}, T_{0}+\varepsilon\left[; H_{D}^{1, q}\right), \\
\zeta \in W_{0}^{1, r}(] T_{0}, T_{0}+\varepsilon\left[; H_{\Omega}^{-1, q}\right) \cap L^{r}(] T_{0}, T_{0}+\varepsilon\left[; H^{1, q}\right)
\end{gathered}
$$

of (3.20). Choosing $T_{0}=0$, the corresponding solution coincides with that of Definition 3.3 with $T=\varepsilon$. The property $\theta \in L^{r}(] 0, \varepsilon\left[; H^{1, q}\right)$ ensures the existence of a point $t \in] \varepsilon / 2, \varepsilon]$ such that $\theta(t) \in H^{1, q}(\Omega) \hookrightarrow L^{\infty}(\Omega)$. Hence, we may start once more, i.e., consider (3.20), this time with $T_{0}=t$. By the contractivity of $\mathcal{K G}$, we again obtain a unique solution of (3.20) on ]t, $t+\varepsilon$ [, which together with the solution on $] 0, t[$ represents the solution of (3.9) and (3.10) on $] 0, t+\varepsilon[$. Finally, repeating this argument yields the unique existence of a solution according to Definition 3.3 on $] 0, T$. Furthermore, part (iii) of Theorem 3.5 follows from Lemma 3.17(iii) and (3.19), and part (iv) is obtained from Lemma 3.20(ii).

4. Existence of an optimal control. The existence of a (globally) optimal control for problem $(\mathrm{P})$ can be shown only under an additional hypothesis on the feasible set; see Assumption 4.4 below. Nevertheless, let us already point out here that this assumption is not necessary for the upcoming analysis, addressing first-order necessary conditions for $(\mathrm{P})$; cf. Remark 4.5. Since the state equation is nonlinear, we can naturally not expect uniqueness of an optimal solution. We start with the definition of the state space

DeFinition 4.1. Let $q$ be the real number from section 3 , hence $q \in] 2, \min \left\{\tilde{q}_{0}, q_{1}\right\}[$, while $r$ satisfies $2 q /(q-2)<r \leq \infty$. Then the state space is defined by

$$
Y:=W^{1, r}(] 0, T\left[; H_{\Omega}^{-1, q}\right) \cap L^{r}\left(S ; H^{1, q}\right)
$$

and thus coincides with the space given in Definition 3.3.

DeFINITION 4.2. Based on Theorem 3.5, we introduce the control-to-state operators $\mathcal{S}: L^{\infty}(] 0, T\left[; L^{2}\left(\Gamma_{N}\right)\right) \rightarrow Y \times L^{\infty}(] 0, T\left[; H_{D}^{1, q}\right), \mathcal{S}_{1}: L^{\infty}(] 0, T\left[; L^{2}\left(\Gamma_{N}\right)\right) \rightarrow Y$, and $\mathcal{S}_{2}: L^{\infty}(] 0, T\left[; L^{2}\left(\Gamma_{N}\right)\right) \rightarrow L^{\infty}(] 0, T\left[; H_{D}^{1, q}\right)$ by

$$
\mathcal{S}(u)=\left(\begin{array}{l}
\mathcal{S}_{1}(u) \\
\mathcal{S}_{2}(u)
\end{array}\right)=\left(\begin{array}{l}
\theta(u) \\
\varphi(u)
\end{array}\right),
$$

where $\theta(u)$ and $\varphi(u)$ denote the solution of (1.1)-(1.7) associated with $u$ in the sense of Definition 3.3. We point out that, in all of what follows, $\mathcal{S}$ is sometimes used with 
different ranges, for simplicity also denoted by $\mathcal{S}$. Using $\mathcal{S}$, we define the reduced objective functional $j: L^{\infty}(] 0, T\left[; L^{2}\left(\Gamma_{N}\right)\right) \rightarrow \mathbb{R}$ by

$$
j(u):=J\left(\mathcal{S}_{1}(u), \mathcal{S}_{2}(u), u\right),
$$

where $J$ is the objective functional of $(\mathrm{P})$.

Definition 4.3. A function $u \in L^{\infty}(] 0, T\left[; L^{2}\left(\Gamma_{N}\right)\right)$ is called feasible for $(\mathrm{P})$ if it satisfies $0 \leq u(x, t) \leq u_{\max }(x, t)$ a.e. in $Q$ and $\mathcal{S}_{1}(u)(x, t) \leq \theta_{\max }(x, t)$ a.e. in $Q$. Moreover, the set $U_{a d}$ is defined by

$$
U_{a d}:=\left\{u \in L^{\infty}(] 0, T\left[, L^{2}\left(\Gamma_{N}\right)\right): 0 \leq u(x, t) \leq u_{\max }(x, t) \text { a.e. in } \Sigma_{N}\right\} .
$$

To show the existence of a globally optimal control, we need an additional assumption, which is stated next.

Assumption 4.4. Let $K$ be a given compact subset of $L^{\infty}(] 0, T\left[; H_{D}^{-1, q}\right)$. Instead of $(\mathrm{P})$, we consider the problem

$$
\left.\begin{array}{rl}
\text { minimize } & j(u) \\
\text { subject to } & \mathcal{S}_{1}(u)(x, t) \leq \theta_{\max }(x, t) \quad \text { a.e. in } Q \\
\text { and } & u \in K_{a d},
\end{array}\right\}
$$

where $K_{a d}=U_{a d} \cap K$.

Remark 4.5. We point out that the restriction of the set of controls to a compact subset of $L^{\infty}(] 0, T\left[; H_{D}^{-1, q}\right)$ is necessary only to show existence of a globally optimal solution. It is not needed for the derivation of first-order necessary optimality conditions for (locally) optimal solutions as carried out in section 6 . Thus we only require this additional assumption within the scope of this section.

Before we are in the position to prove an existence result for $(\tilde{\mathrm{P}})$, the continuity of the fixed point mapping $\mathcal{K G}$ is addressed in the next lemma.

LEmma 4.6. Let $\mathcal{K G}$ be defined as in the previous section (cf. (3.18)), and indicate the additional dependence of $\mathcal{K G}$ on the control $u$ by $(\mathcal{K G})_{u}$. Then, the following hold:

(i) The mapping

$$
L^{\infty}(] 0, T\left[; H^{-1, q}\right) \times L^{\infty}\left(\left[0, T\left[; L^{\infty}(\Omega)\right) \ni(u, \zeta) \mapsto(\mathcal{K G})_{u}(\zeta)\right.\right.
$$

takes its values in $C([0, T] \times \bar{\Omega})$ and is continuous.

(ii) If $M$ is a bounded set in $L^{\infty}(] 0, T\left[; H^{-1, q}\right)$, then the set

$$
\left\{(\mathcal{K G})_{u}(\zeta): u \in M, \zeta \in L^{\infty}\left(\left[0, T\left[; L^{\infty}(\Omega)\right)\right\}\right.\right.
$$

is bounded in $W_{0}^{1, r}(] 0, T\left[; H_{\Omega}^{-1, q}\right) \cap L^{r}(] 0, T\left[; H^{1, q}\right)$ and, by embedding, even precompact in $C([0, T] \times \bar{\Omega})$.

Proof. The assertions are mainly obtained by slightly extending the considerations in Lemma 3.9. For the convenience of the reader, we briefly sketch the arguments leading to the first assertion. The continuity of $(\zeta, u) \mapsto \varphi$ is easily seen analogously 
to part (ii) of Lemma 3.9:

$$
\begin{aligned}
\left\|\left(\nabla \cdot \sigma\left(\zeta_{1}+\iota_{0}\right)(t) \nabla\right)^{-1} u_{1}(t)-\left(\nabla \cdot \sigma\left(\zeta_{2}+\iota_{0}\right)(t) \nabla\right)^{-1} u_{2}(t)\right\|_{H_{D}^{1, q}} \\
\leq\left\|\left[\left(\nabla \cdot \sigma\left(\zeta_{1}+\iota_{0}\right)(t) \nabla\right)^{-1}-\left(\nabla \cdot \sigma\left(\zeta_{2}+\iota_{0}\right)(t) \nabla\right)^{-1}\right] u_{2}(t)\right\|_{H_{D}^{1, q}} \\
\quad+\left\|\left(\nabla \cdot \sigma\left(\zeta_{1}+\iota_{0}\right)(t) \nabla\right)^{-1}\left(u_{1}(t)-u_{2}(t)\right)\right\|_{H_{D}^{1, q}} \\
\leq\left\|\left(\nabla \cdot \sigma\left(\zeta_{1}+\iota_{0}\right)(t) \nabla\right)^{-1}\right\|_{\mathcal{B}\left(H_{D}^{-1, q} ; H_{D}^{1, q}\right)}\left\|\left(\nabla \cdot \sigma\left(\zeta_{2}+\iota_{0}\right)(t) \nabla\right)^{-1}\right\|_{\mathcal{B}\left(H_{D}^{-1, q} ; H_{D}^{1, q}\right)} \\
\quad L_{\sigma}\left\|u_{2}(t)\right\|_{H_{D}^{-1, q}\left\|\zeta_{1}(t)-\zeta_{2}(t)\right\|_{L^{\infty}(\Omega)}} \\
\quad+\left\|\left(\nabla \cdot \sigma\left(\zeta_{1}+\iota_{0}\right)(t) \nabla\right)^{-1}\right\|_{\mathcal{B}\left(H_{D}^{-1, q} ; H_{D}^{1, q}\right)}\left\|u_{1}(t)-u_{2}(t)\right\|_{H_{D}^{-1, q}} .
\end{aligned}
$$

Similarly to the proof of Lemma 3.11 , the continuity of $\mathcal{G}$ w.r.t. $(u, \zeta)$ is derived. Finally, the boundedness of $\mathcal{K}$ implies the assertion.

TheOREm 4.7. Let Assumptions 2.1-2.4 and 4.4 be fulfilled, and assume that there is at least one feasible control. Then there exists an optimal solution of problem $(\tilde{\mathrm{P}})$.

Remark 4.8. We point out that the assumption of existence of a feasible control in the above theorem is fulfilled in many practically relevant situations. If, for instance, $K$ is given as in Remark 4.9 below, $\theta_{0}=\theta_{l} \equiv 290 \mathrm{~K}$, and $\theta_{\max }(x, t) \geq 290 \mathrm{~K}$ a.e. in $\Omega$ (cf. the numerical example in section 7 ), then $u \equiv 0$ is a feasible control, since we clearly have $\mathcal{S}_{1}(0)(x) \equiv 290 \mathrm{~K}$ in this case.

Proof of Theorem 4.7. Since there is a feasible control and the objective functional $J$ is clearly bounded from below, there is a minimizing sequence of feasible controls, denoted by $\left\{u_{n}\right\}$. According to the compactness of $K \subset L^{\infty}(] 0, T\left[; H_{D}^{-1, q}\right)$, there is a subsequence $\left\{u_{k}\right\}$ converging to an element $\bar{u} \in K$ in $L^{\infty}(] 0, T\left[; H_{D}^{-1, q}\right)$. First we show that $\bar{u}$ can be interpreted as a function in $U_{a d}$. To this end, observe that, by possibly passing to a further subsequence, we may suppose that $\left\{u_{k}\right\}$ converges weakly in $L^{2}(] 0, T\left[\times \Gamma_{N}\right)$ to an element $w$. Since $U_{a d}$ is closed and convex, it is weakly closed, giving in turn $w \in U_{a d}$. Moreover, due to $q<4$, the trace operator maps $H_{D}^{1, q^{\prime}}$ continuously into $L^{2}(\partial \Omega) \hookrightarrow L^{2}\left(\Gamma_{N}\right)$, such that $w$ uniquely defines an element $\tilde{w} \in L^{2}(] 0, T\left[; H_{D}^{-1, q}\right)$ via

$$
\langle\tilde{w}, v\rangle_{L^{2}\left(H^{-1, q}\right), L^{2}\left(H^{1, q^{\prime}}\right)}:=\int_{0}^{T} \int_{\Gamma_{N}} w(x, t) \tau_{N}(v)(x, t) d s d t, \quad v \in L^{2}(] 0, T\left[; H_{D}^{1, q^{\prime}}\right),
$$

where $\tau_{N}: H_{D}^{1, q^{\prime}} \rightarrow L^{2}\left(\Gamma_{N}\right)$ is the trace operator on $\Gamma_{N}$. Now, in view of the weak convergence of $\left\{u_{k}\right\}$ in $L^{2}(] 0, T\left[\times \Gamma_{N}\right)$ and its strong convergence in $L^{2}(] 0, T\left[; H_{D}^{-1, q}\right)$, we clearly obtain $\tilde{w}=\bar{u}$ in $L^{2}(] 0, T\left[; H_{D}^{-1, q}\right)$. Hence, $\bar{u}$ is indeed represented by the $L^{2}(] 0, T\left[\times \Gamma_{N}\right)$-function $w$. We are thus allowed to identify $\bar{u} \in K$ and $w \in U_{a d}$ as an element $\bar{u} \in K_{a d}$.

Next, we prove that the state system (3.9)-(3.10) is satisfied in the limit. As depicted at the end of the previous section, for given $u \in L^{\infty}\left(\left[0, T\left[; L^{2}\left(\Gamma_{N}\right)\right),(3.9)-\right.\right.$ (3.10) is fulfilled iff $\zeta$ is a fixed point of the mapping $\mathcal{K G}$ and $\varphi$ is defined via the elliptic equation (3.10). Denoting the fixed point of the mapping $(\mathcal{K G})_{u_{k}}$ by $\zeta_{k}$, part (ii) of Lemma 4.6 assures the existence of a subsequence $\left\{\zeta_{l}\right\}$ such that the sequence $\left\{(\mathcal{K G})_{u_{l}}\left(\zeta_{l}\right)\right\}=\left\{\zeta_{l}\right\}$ converges in $C([0, T] \times \bar{\Omega})$ towards an element $\bar{\zeta}$. Hence, because of $(\mathcal{K G})_{u_{l}}\left(\zeta_{l}\right)-\zeta_{l}=0$, part (i) of Lemma 4.6 yields $(\mathcal{K G})_{\bar{u}}(\bar{\zeta})-\bar{\zeta}=0$. Therefore, $\bar{\zeta}$ provides a solution of (3.9)-(3.10), belonging to the limit control $\bar{u}$.

In addition, since $\left\{\zeta_{l}\right\}$ converges to $\bar{\zeta}$ in $C([0, T] \times \bar{\Omega}), \bar{\theta}:=\bar{\zeta}+\iota_{0}$ also satisfies the state constraints; i.e., $\bar{\theta}(x, t) \leq \theta_{\max }(x, t)$ a.e. in $Q$. Finally, since the objective 
is clearly weakly lower semicontinuous, $(\bar{\theta}, \bar{\varphi}, \bar{u})$ represents an optimal solution of $(\tilde{\mathrm{P}})$.

Remark 4.9. Let us give an example for the set $K$ from Assumption 4.4. Assume that a (fixed) partition of $[0, T]$ into subintervals is given, defined by the points $0=$ $t_{1}<t_{2}<\cdots<t_{n+1}=T$. Additionally, we fix the two constants $\alpha, C>0$. Then, the set $K$ from Assumption 4.4 is defined by

$$
K:=\left\{u \in L^{\infty}(] 0, T\left[; H_{D}^{-1, q}\right):\left\|\left.u\right|_{\left(t_{i}, t_{i+1}\right)}\right\|_{C^{\alpha}\left(t_{i}, t_{i+1} ; H_{D}^{-1, q}\right)} \leq C \forall i=1,2, \ldots, n\right\} .
$$

Considering a sequence $\left\{v_{k}\right\}$ from $K$, it follows from [29, sec. 6, Thm. 3] that the restrictions of the sequence to each of the intervals $] t_{j}, t_{j+1}[$ admit a subsequence which converges in $L^{\infty}(] t_{j}, t_{j+1}\left[; H_{D}^{-1, q}\right)$. Thus, one can extract a subsequence which converges as a whole in $L^{\infty}(] 0, T\left[; H_{D}^{-1, q}\right)$. Moreover, it is easily shown that the limit is again contained in $K$, so that $K$ satisfies the conditions in Assumption 4.4.

This example shows that the setting of Theorem 4.7 allows us to consider controls which are discontinuous in time. This is essential in the presence of state constraints, since the optimality system does not imply continuity of optimal controls in this case; see (6.47).

5. Analysis of the linearized state system. For the derivation of first-order optimality conditions, it is essential to show the Fréchet-differentiability of the controlto-state operator, mapping $u$ to $\theta$ (see section 6.1 below). In preparation for a corresponding theorem, we now consider the following linearized version of the thermistor problem (1.1)-(1.7):

$$
\begin{aligned}
\partial_{t} \theta^{\prime}-\operatorname{div}\left(\kappa \nabla \theta^{\prime}\right) & =\left(\sigma^{\prime}(\theta) \theta^{\prime} \nabla \varphi\right) \cdot \nabla \varphi+2(\sigma(\theta) \nabla \varphi) \cdot \nabla \varphi^{\prime}+f_{1} & & \text { in } Q, \\
\nu \cdot \kappa \nabla \theta^{\prime}+\alpha \theta^{\prime} & =f_{2} \quad & & \text { on } \partial \Omega \times] 0, T[, \\
\theta^{\prime}\left(T_{0}\right) & =\theta_{0}^{\prime} & & \text { in } \Omega, \\
-\operatorname{div}\left(\sigma(\theta) \nabla \varphi^{\prime}\right) & =\operatorname{div}\left(\sigma^{\prime}(\theta) \theta^{\prime} \nabla \varphi\right)+g_{1} & & \text { in } Q, \\
\nu \cdot \sigma(\theta) \nabla \varphi^{\prime} & =-\nu \cdot \sigma^{\prime}(\theta) \theta^{\prime} \nabla \varphi+g_{2} & & \text { on } \left.\partial \Omega \backslash \Gamma_{D} \times\right] 0, T[, \\
\varphi^{\prime} & =0 & & \text { on } \left.\Gamma_{D} \times\right] 0, T[,
\end{aligned}
$$

with given functions $\theta, \varphi, \theta_{0}^{\prime}, f_{i}$, and $g_{i}, i=1,2$, which are specified in the subsequent section (cf. Assumption 5.1). Later, $\theta$ and $\varphi$ will be the solution of the nonlinear state system (1.1)-(1.7) associated with a reference control. In the following, we will show that (5.1)-(5.6) admit a unique solution $\left(\theta^{\prime}, \varphi^{\prime}\right)$ which is Hölder continuous in space and time. This result is then used to establish Fréchet-differentiability of the solution operator associated with (1.1)-(1.7); see section 6.1.

5.1. Additional assumptions and existence result. Beside Assumptions 2.1 and 2.3, we need the following assumptions for the discussion of (5.1)-(5.6), in particular an additional hypothesis on $\sigma$.

Assumption 5.1. In addition to Assumptions 2.1 and 2.3, the quantities in (5.1)(5.6) satisfy the following:

(i) $\theta_{0}^{\prime} \in L^{\infty}(\Omega)$.

(ii) $\theta$ and $\varphi$ are fixed functions in $L^{\infty}(] 0, T\left[; L^{\infty}(\Omega)\right)$ and $L^{\infty}(] 0, T\left[; H_{D}^{1, q}(\Omega)\right)$ with $\left.q \in] 2, \min \left\{\tilde{q}_{0}, q_{1}\right\}\right]$, where $\tilde{q}_{0}$ and $q_{1}$ are the numbers from Theorems 3.18 and 3.7, respectively (such that $q \in] 2,4[$ ).

(iii) The functions $f_{1}, f_{2}, g_{1}$, and $g_{2}$ define elements of $L^{s}(] 0, T\left[, H_{\Omega}^{-1, q}(\Omega)\right)$ and $L^{s}(] 0, T\left[, H_{D}^{-1, q}(\Omega)\right)$, respectively, where $\left.\left.s \in\right] q /(q-2), \infty\right]$. 
(iv) Each component of the matrix $\sigma=\sigma(x, \theta)$ is continuously differentiable w.r.t. $\theta$ for almost all $x \in \Omega$, and there is a constant $C>0$ such that $\left\|\sigma^{\prime}(x, 0)\right\|_{\mathcal{B}\left(\mathbb{R}^{2}\right)} \leq C$. Furthermore, the derivative of $\sigma$ is locally Lipschitz continuous; i.e., for every real number $M>0$, there exists a constant $L(M)>0$ such that

$$
\left\|\sigma^{\prime}(x, \tilde{\theta})-\sigma^{\prime}(x, \theta)\right\|_{\mathcal{B}\left(\mathbb{R}^{2}\right)} \leq L(M)|\tilde{\theta}-\theta|
$$

for all $\tilde{\theta}, \theta \in[-M, M]$ and almost all $x \in \Omega$.

Similarly to Remark 3.2, one verifies that Assumption 5.1 (iii) is fulfilled if $f_{1}, g_{1} \in$ $L^{s}\left(S ; L^{\rho_{1}}(\Omega)\right), f_{2} \in L^{s}\left(S ; L^{\rho_{2}}(\partial \Omega)\right)$, and $g_{2} \in L^{s}\left(S ; L^{\rho_{2}}\left(\partial \Omega \backslash \Gamma_{D}\right)\right)$ hold true with $\rho_{1} \geq 2 q /(q+2)$ and $\rho_{2} \geq q / 2$. As before, we denote the associated functionals by $\tilde{f}_{i}$ and $\tilde{g}_{i}, i=1,2$, and define $\tilde{f}:=\tilde{f}_{1}+\tilde{f}_{2}$ and $\tilde{g}:=\tilde{g}_{1}+\tilde{g}_{2}$. Furthermore, Assumption 5.1 implies that the Nemytskii-operator associated with $\sigma^{\prime}$ is continuous from $L^{\infty}(] 0, T\left[; L^{\infty}(\Omega)\right)$ to $L^{\infty}(] 0, T\left[; L^{\infty}\left(\Omega ; \mathcal{B}\left(\mathbb{R}^{2}\right)\right)\right)$ and that there holds

$$
\left\|\sigma^{\prime}(\theta)\right\|_{L^{\infty}(] 0, T\left[; L^{\infty}\left(\Omega ; \mathcal{B}\left(\mathbb{R}^{2}\right)\right)\right)} \leq C+L\left(\|\theta\|_{L^{\infty}(] 0, T\left[; L^{\infty}(\Omega)\right)}\right)\|\theta\|_{L^{\infty}(] 0, T\left[; L^{\infty}(\Omega)\right)} .
$$

Similarly to section 3.1 , we set $\iota_{T_{0}}^{\prime}(t)=e^{-\left(t-T_{0}\right) K} \theta_{0}^{\prime}, t \geq T_{0}$, such that $\iota_{T_{0}}^{\prime}$ exhibits the same properties as $\iota_{T_{0}}$, in particular Lemma 3.20.

Definition 5.2. A pair $\left(\theta^{\prime}, \varphi^{\prime}\right)$ is considered as solution of (5.1)-(5.6) if there exist indices $q$ and s satisfying the conditions in Assumption 5.1(ii) and (iii) such that $\varphi^{\prime}$ and $\zeta^{\prime}:=\theta^{\prime}-\iota_{0}^{\prime}$ satisfy

$$
\begin{aligned}
& \varphi^{\prime} \in L^{s}(] 0, T\left[; H_{D}^{1, q}\right), \\
& \zeta^{\prime} \in \begin{cases}W_{0}^{1, s}(] 0, T\left[; H_{\Omega}^{-1, q}\right) \cap L^{s}(] 0, T\left[; H^{1, q}\right) & \text { if } s<\infty, \\
W_{0}^{1, \rho}(] 0, T\left[; H_{\Omega}^{-1, q}\right) \cap L^{\rho}(] 0, T\left[; H^{1, q}\right) \quad \forall \rho<\infty & \text { if } s=\infty,\end{cases}
\end{aligned}
$$

and additionally the operator equations

$$
\begin{gathered}
\partial_{t} \zeta^{\prime}+K \zeta^{\prime}=\left(\sigma^{\prime}(\theta)\left(\zeta^{\prime}+\iota_{0}^{\prime}\right) \nabla \varphi\right) \cdot \nabla \varphi+2 \sigma(\theta) \nabla \varphi \cdot \nabla \varphi^{\prime}+\tilde{f} \\
-\nabla \cdot\left(\sigma(\theta) \nabla \varphi^{\prime}\right)=\nabla \cdot\left(\sigma^{\prime}(\theta)\left(\zeta^{\prime}+\iota_{0}^{\prime}\right) \nabla \varphi\right)+\tilde{g}
\end{gathered}
$$

hold true.

Notice that, due to $H^{1, q}(\Omega) \hookrightarrow L^{\infty}(\Omega), q>2$, we have $\sigma^{\prime}(\theta(t)) \theta^{\prime}(t) \in L^{\infty}(\Omega)$ for almost all $t \in] 0, T\left[\right.$ such that $\nabla \cdot \sigma^{\prime}(\theta(t)) \theta^{\prime}(t) \nabla: H_{D}^{1, q} \rightarrow H_{D}^{-1, q}$ is defined as in (3.2).

THEOREM 5.3.

(i) There is a solution of (5.1)-(5.6) in the sense of Definition 5.2.

(ii) This solution is unique.

(iii) If $s>2 q /(q-2)$ and $\theta_{0}^{\prime} \in H^{\varsigma, q}, \varsigma>\frac{2}{q}$, then $\theta^{\prime} \in C^{\eta}(] 0, T\left[; C^{\eta}(\Omega)\right)$.

5.2. Proof of Theorem 5.3. The proof basically follows the lines of the analysis for the nonlinear state system investigated in section 3. Again, $T_{0}$ and $T_{1}$ are fixed but arbitrary numbers satisfying $0 \leq T_{0}<T_{1}<\infty$, and $\left.S=\right] T_{0}, T_{1}[$. We start with the investigation of the elliptic equation (5.11). Similar to Lemma 3.9, we find the following.

Lemma 5.4. Let $\gamma$ be defined by $\gamma:=\frac{2 q}{q-2}$, and $\tilde{g}$ be given in $L^{s}\left(S ; H_{D}^{-1, q}\right)$. Then, the affine linear mapping $\mathcal{H}$ which assigns to every $\zeta^{\prime} \in L^{s}\left(S ; L^{\gamma}\right)$ the solution $\varphi^{\prime}$ of

$$
-\nabla \cdot\left(\sigma(\theta) \nabla \varphi^{\prime}\right)=\nabla \cdot\left(\sigma^{\prime}(\theta)\left(\zeta^{\prime}+\iota_{T_{0}}^{\prime}\right) \nabla \varphi\right)+\tilde{g}
$$

Copyright $@$ by SIAM. Unauthorized reproduction of this article is prohibited. 
is Lipschitz continuous from $L^{s}\left(S ; L^{\gamma}\right)$ to $L^{s}\left(S ; H_{D}^{1,2}\right)$. Moreover, the associated Lipschitz constant depends neither on $S$ nor on $\theta_{0}^{\prime}$.

Proof. First, we find $1 / \gamma+1 / q=1 / 2$ such that $\iota_{T_{0}}^{\prime} \in L^{\infty}\left(S ; L^{\infty}\right)$ (by Lemma 3.20), $\zeta^{\prime} \in L^{s}\left(S ; L^{\gamma}\right)$, and $\varphi \in L^{\infty}\left(S ; H_{D}^{1, q}\right)$ imply $\left(\zeta^{\prime}+\iota_{T_{0}}^{\prime}\right) \nabla \varphi \in L^{s}\left(S ; L^{2}\right)^{2}$. Hence, due to Theorem $3.7,(5.12)$ admits a unique solution in $L^{s}\left(S ; H_{D}^{1,2}\right)$ for every $\tilde{g} \in$ $L^{s}\left(S ; H_{D}^{-1, q}\right)$, since $\sigma^{\prime}(\theta) \in L^{\infty}\left(S ; L^{\infty}\left(\Omega ; \mathcal{B}\left(\mathbb{R}^{2}\right)\right)\right)$ according to (5.7). Moreover, one has

$$
\begin{aligned}
& \left\|\tilde{\varphi}^{\prime}-\varphi^{\prime}\right\|_{L^{s}\left(S ; H_{D}^{1,2}\right)} \\
& \quad \leq \|-(\nabla \cdot \sigma(\theta)) \nabla)^{-1}\left\|_{L^{\infty}\left(S ; \mathcal{B}\left(H_{D}^{-1,2} ; H_{D}^{1,2}\right)\right)}\right\| \nabla \cdot \sigma^{\prime}(\theta)\left(\tilde{\zeta}^{\prime}-\zeta^{\prime}\right) \nabla \varphi \|_{L^{s}\left(H_{D}^{-1,2}\right)} .
\end{aligned}
$$

For the latter norm, we find

$$
\begin{aligned}
\left\|\nabla \cdot \sigma^{\prime}(\theta) a\left(\tilde{\zeta}^{\prime}-\zeta^{\prime}\right) \nabla \varphi\right\|_{H_{D}^{-1,2}} & =\sup _{\|v\|_{H_{D}^{1,2}}^{1,1}}\left|\int_{\Omega} \sigma^{\prime}(\theta)\left(\tilde{\zeta}^{\prime}-\zeta^{\prime}\right) \nabla \varphi \cdot \nabla v d x\right| \\
& \leq \sup _{\|v\|_{H_{D}^{1,2}=1}}\left\|\sigma^{\prime}(\theta)\right\|_{L^{\infty}}\|\varphi\|_{H_{D}^{1, q}}\left\|\left(\tilde{\zeta}^{\prime}-\zeta^{\prime}\right) \nabla v\right\|_{L^{q /(q-1)}} \\
& \leq\left\|\sigma^{\prime}(\theta)\right\|_{L^{\infty}}\|\varphi\|_{H_{D}^{1, q}}\left\|\tilde{\zeta}^{\prime}-\zeta^{\prime}\right\|_{L^{\gamma}},
\end{aligned}
$$

with $\left\|\sigma^{\prime}(\theta)\right\|_{L^{\infty}}:=\left\|\sigma^{\prime}(\theta)\right\|_{L^{\infty}\left(\Omega ; \mathcal{B}\left(\mathbb{R}^{2}\right)\right)}$, which is also used in what follows. Together with our assumptions on $\sigma, \varphi$, and $\theta$, and Theorem 3.7(ii), this implies the assertion.

Now, we turn to the right-hand side of (5.10).

Lemma 5.5. The mapping $\mathcal{Q}: L^{s}\left(S ; L^{\gamma}\right) \rightarrow L^{s}\left(S ; H_{\Omega}^{-1, q}\right)$ with $\gamma$ as in Lemma 5.4, given by

$$
\mathcal{Q}: \zeta^{\prime} \mapsto\left(\sigma^{\prime}(\theta)\left(\zeta^{\prime}+\iota_{T_{0}}^{\prime}\right) \nabla \varphi\right) \cdot \nabla \varphi+2 \sigma(\theta) \nabla \varphi \cdot \nabla \mathcal{H}\left(\zeta^{\prime}\right)+\tilde{f}
$$

is Lipschitzian with a Lipschitz constant independent of $S$ and $\theta_{0}^{\prime}$.

Proof. Using Hölder's inequality twice yields for the first part in the image of $\mathcal{Q}$

$$
\begin{aligned}
\left\|\left(\sigma^{\prime}(\theta)\left(\tilde{\zeta}^{\prime}-\zeta^{\prime}\right) \nabla \varphi\right) \cdot \nabla \varphi\right\|_{H_{\Omega}^{-1, q}} & \leq \sup _{\|v\|_{H^{1, q^{\prime}}=1}}\left\|\sigma^{\prime}(\theta)\right\|_{L^{\infty}}\|(\nabla \varphi)\|_{L^{q}}^{2}\left\|\left(\tilde{\zeta}^{\prime}-\zeta^{\prime}\right) v\right\|_{L^{(q-2) / q}} \\
& \leq \sup _{\|v\|_{H^{1, q^{\prime}}=1}}\left\|\sigma^{\prime}(\theta)\right\|_{L^{\infty}}\|\varphi\|_{H_{D}^{1, q}}^{2}\left\|\tilde{\zeta}^{\prime}-\zeta^{\prime}\right\|_{L^{\gamma}}\|v\|_{L^{2 q /(q-2)}} \\
& \leq c\left\|\sigma^{\prime}(\theta)\right\|_{L^{\infty}}\|\varphi\|_{H_{D}^{1, q}}^{2}\left\|\tilde{\zeta}^{\prime}-\zeta^{\prime}\right\|_{L^{\gamma}},
\end{aligned}
$$

where we have used the continuous embedding $H^{1, q^{\prime}}(\Omega) \hookrightarrow L^{2 q /(q-2)}(\Omega)$ for the last estimate. The second part is estimated by

$$
\begin{aligned}
\left\|(\sigma(\theta) \nabla \varphi) \cdot \nabla\left(\mathcal{H}\left(\tilde{\zeta}^{\prime}\right)-\mathcal{H}\left(\zeta^{\prime}\right)\right)\right\|_{H_{\Omega}^{-1, q}} & \leq \sup _{\|v\|_{H^{1, q^{\prime}}=1}}\|\sigma(\theta)\|_{L^{\infty}}\|\varphi\|_{H_{D}^{1, q}}\left\|\nabla\left(\tilde{\varphi}^{\prime}-\varphi^{\prime}\right) v\right\|_{L^{q /(q-1)}} \\
& \leq \sup _{\|v\|_{H^{1, q^{\prime}}=1}}\|\sigma(\theta)\|_{L^{\infty}}\|\varphi\|_{H_{D}^{1, q}}\left\|\tilde{\varphi}^{\prime}-\varphi^{\prime}\right\|_{H_{D}^{1,2}}\|v\|_{L^{2 q /(2-2)}} \\
& \leq\|\sigma(\theta)\|_{L^{\infty}}\|\varphi\|_{H_{D}^{1, q}}\left\|\tilde{\zeta}^{\prime}-\zeta^{\prime}\right\|_{L^{\gamma}},
\end{aligned}
$$

Copyright (c) by SIAM. Unauthorized reproduction of this article is prohibited. 
where Lemma 5.4 gives the latter estimate. Based on these estimates, we obtain

$$
\begin{aligned}
& \left\|\mathcal{Q}\left(\tilde{\zeta}^{\prime}\right)-\mathcal{Q}\left(\zeta^{\prime}\right)\right\|_{L^{s}\left(S ; H_{\Omega}^{-1, q}\right)} \\
& \leq \int_{S}\left(\left\|\left(\sigma^{\prime}(\theta)\left(\tilde{\zeta}^{\prime}-\zeta^{\prime}\right) \nabla \varphi\right) \cdot \nabla \varphi\right\|_{H_{\Omega}^{-1, q}}+2\left\|(\sigma(\theta) \nabla \varphi) \cdot \nabla\left(\tilde{\varphi}^{\prime}-\varphi^{\prime}\right)\right\|_{H_{\Omega}^{-1, q}}\right)^{s} d t^{1 / s} \\
& \leq\left(\left\|\sigma^{\prime}(\theta)\right\|_{L^{\infty}\left(S ; L^{\infty}\right)}\|\varphi\|_{L^{\infty}\left(S ; H_{D}^{1, q}\right)}^{2}\right. \\
& \left.\quad+2\|\sigma(\theta)\|_{L^{\infty}\left(S ; L^{\infty}\right)}\|\varphi\|_{L^{\infty}\left(S ; H_{D}^{1, q}\right)}\right)\left\|\tilde{\zeta}^{\prime}-\zeta^{\prime}\right\|_{L^{s}\left(S ; L^{\gamma}\right)} .
\end{aligned}
$$

Thanks to our assumptions on $\sigma, \theta$, and $\varphi$, the expression in the brackets does not depend on $S$ or $\theta_{0}^{\prime}$.

Similarly to the operator $\mathcal{K G}$ in section 3 , we now consider the combined mapping:

$$
\mathcal{W}:=\mathcal{E}_{\infty} \mathcal{L} \mathcal{Q}: L^{s}\left(S ; L^{\gamma}\right) \rightarrow L^{\infty}\left(S ; L^{\gamma}\right)
$$

where $\mathcal{L}: L^{s}\left(S ; H_{\Omega}^{-1, q}\right) \rightarrow W_{0}^{1, s}\left(S ; H_{\Omega}^{-1, q}\right) \cap L^{s}\left(S ; H^{1, q}\right)$ is defined as in Lemma 3.16. Moreover, $\mathcal{E}_{\infty}$ denotes the embedding of $W_{0}^{1, s}\left(S ; H_{\Omega}^{-1, q}\right) \cap L^{s}\left(S ; H^{1, q}\right)$ in $L^{\infty}\left(S ; L^{\gamma}\right)$, which is well defined since $W_{0}^{1, s}\left(S ; H_{\Omega}^{-1, q}\right) \cap L^{s}\left(S ; H^{1, q}\right) \hookrightarrow C\left(\bar{S} ; H^{2 \eta-1, q}\right), \eta<1-1 / s$, by (3.19), and $H^{2 \eta-1, q} \hookrightarrow L^{\gamma}$ for $s>q /(q-2)$, which is ensured by Assumption 5.1(iii). Clearly, due to the above lemmas and the results of section 3, in particular Lemma 3.16 and Lemma 3.17, $\mathcal{W}$ is Lipschitz continuous from $L^{s}\left(S ; L^{\gamma}\right)$ to $L^{\infty}\left(S ; L^{\gamma}\right)$ with a Lipschitz constant $L_{\mathcal{W}}$ independent of $\theta_{0}^{\prime}$ and $S$. Furthermore, if we consider the operator $\mathcal{W}_{s}=\mathcal{E}_{s} \mathcal{W}$, where $\mathcal{E}_{s}: L^{\infty}\left(S ; L^{\gamma}\right) \rightarrow L^{s}\left(S ; L^{\gamma}\right)$ denotes associated embedding, we obtain for its Lipschitz constant

$$
\begin{aligned}
\left\|\mathcal{W}_{s}\left(\tilde{\zeta}^{\prime}\right)-\mathcal{W}_{s}\left(\zeta^{\prime}\right)\right\|_{L^{s}\left(S ; L^{\gamma}\right)} & \leq \int_{S}\left\|\left(\mathcal{W}_{s}\left(\tilde{\zeta}^{\prime}\right)-\mathcal{W}_{s}\left(\zeta^{\prime}\right)\right)(t)\right\|_{L^{\gamma}}^{s} d t^{1 / s} \\
& \leq\left|T_{1}-T_{0}\right|^{1 / s}\left\|\mathcal{W}\left(\tilde{\zeta}^{\prime}\right)-\mathcal{W}\left(\zeta^{\prime}\right)\right\|_{L^{\infty}(S ; L \gamma} \\
& \leq L_{\mathcal{W}}\left|T_{1}-T_{0}\right|^{1 / s}\left\|\tilde{\zeta}^{\prime}-\zeta^{\prime}\right\|_{L^{s}\left(S ; L^{\gamma}\right)}
\end{aligned}
$$

such that $\mathcal{W}_{s}: L^{s}\left(S ; L^{\gamma}\right) \rightarrow L^{s}\left(S ; L^{\gamma}\right)$ is contractive for sufficiently small $T_{1}-T_{0}$. The rest of the proof is completely analogous to the theory in the nonlinear case: By construction, the fixed point equation

$$
\zeta^{\prime}=\mathcal{W}_{s} \zeta^{\prime} \quad \text { in } L^{s}\left(S ; L^{\gamma}\right)
$$

is equivalent to the operator equation

$$
\begin{gathered}
\partial_{t} \zeta^{\prime}+K \zeta^{\prime}=\left(\sigma^{\prime}(\theta)\left(\zeta^{\prime}+\iota_{T_{0}}^{\prime}\right) \nabla \varphi\right) \cdot \nabla \varphi+2(\sigma(\theta) \nabla \varphi) \cdot \nabla \varphi^{\prime}+\tilde{f} \\
-\nabla \cdot\left(\sigma(\theta) \nabla \varphi^{\prime}\right)=\nabla \cdot\left(\sigma^{\prime}(\theta)\left(\zeta^{\prime}+\iota_{T_{0}}^{\prime}\right) \nabla \varphi\right)+\tilde{g}
\end{gathered}
$$

on $] T_{0}, T_{1}$ [. Provided that $T_{1}-T_{0}$ is small enough, Banach's contraction principle again yields the existence of a unique fixed point and consequently a solution,

$$
\begin{gathered}
\varphi^{\prime} \in L^{s}(] T_{0}, T_{1}\left[; H_{D}^{1, q}\right), \\
\zeta^{\prime} \in W_{0}^{1, s}(] T_{0}, T_{1}\left[; H_{\Omega}^{-1, q}\right) \cap L^{s}(] T_{0}, T_{1}\left[; H^{1, q}\right),
\end{gathered}
$$

of (5.13). By the same arguments as in section 3, one can repeat the fixed point technique to obtain a solution on the whole time interval $] 0, T[$. As in the nonlinear case, 
the additional regularity of $\theta^{\prime}$, stated in Theorem 5.3(iii), follows from Lemma 3.17(iii), (3.19), and Lemma 3.20. Notice that Lemma 5.4 just implies $\varphi^{\prime} \in L^{s}\left(S ; H_{D}^{1,2}\right)$. However, similarly to the proof of Lemma 5.4, one obtains

$$
\begin{array}{r}
\left.\left\|\varphi^{\prime}\right\|_{L^{s}\left(S ; H_{D}^{1, q}\right)} \leq \|-(\nabla \cdot \sigma(\theta)) \nabla\right)^{-1} \|_{L^{\infty}\left(S ; \mathcal{B}\left(H_{D}^{-1, q} ; H_{D}^{1, q}\right)\right)} \\
\left\|\nabla \cdot \sigma^{\prime}(\theta) \theta^{\prime} \nabla \varphi+\tilde{g}\right\|_{L^{s}\left(H_{D}^{-1, q}\right)} \\
\leq c\left(\left\|\sigma^{\prime}(\theta)\right\|_{L^{\infty}\left(S ; L^{\infty}\right)}\|\varphi\|_{L^{\infty}\left(S ; H_{D}^{1, q}\right)}\left\|\tilde{\zeta}^{\prime}+\iota_{0}^{\prime}\right\|_{L^{s}\left(S ; L^{\infty}\right)}\right. \\
\left.+\|\tilde{g}\|_{L^{s}\left(S ; H_{D}^{-1, q}\right)}\right),
\end{array}
$$

where we used Theorem 3.7(ii) for the last estimate. Now, by Lemma 3.20(i), we have $\iota_{0}^{\prime} \in L^{\infty}\left(S ; L^{\infty}\right)$. Moreover, due to $q>2, \zeta^{\prime} \in L^{s}\left(S ; H^{1, q}\right)$ implies $\zeta^{\prime} \in L^{s}\left(S ; L^{\infty}\right)$ such that $\varphi^{\prime} \in L^{s}\left(S ; H_{D}^{1, q}\right)$ according to Definition 5.2.

Remark 5.6. Suppose that $(\theta, \varphi)$ is a solution of the nonlinear state system $(1.1)-$ (1.7) in the sense of Definition 3.3. Then, $(\theta, \varphi) \in C([0, T] ; C(\bar{\Omega})) \cap L^{\infty}(] 0, T\left[, H_{D}^{1, q}\right)$ such that Assumption 5.1(ii) is fulfilled. Hence, Theorem 5.3 ensures the existence of a unique solution $\left(\theta^{\prime}, \varphi^{\prime}\right) \in W^{1, s}(] 0, T\left[; H_{\Omega}^{-1, q}\right) \cap L^{s}(] 0, T\left[; H^{1, q}\right) \times L^{s}\left(S ; H_{D}^{1, q}\right)$, $s>q /(q-2)$, for every right-hand side $\tilde{f} \in L^{s}(] 0, T\left[; H_{\Omega}^{-1, q}\right)$ and $\tilde{g} \in L^{s}(] 0, T\left[; H_{D}^{-1, q}\right)$. Moreover, Theorem 5.3 guarantees $\theta^{\prime} \in L^{\infty}(] 0, T\left[; L^{\infty}\right)$, provided that $s>2 q /(q-$ $2)$. If we further suppose that $\tilde{g}$ is more regular, i.e., $\tilde{g} \in L^{\rho}(] 0, T\left[; H_{D}^{-1, q}\right)$ with $\rho>s>2 q /(q-2)$, then an estimate, analogous to (5.14), immediately implies $\varphi^{\prime} \in L^{\rho}(] 0, T\left[; H_{D}^{1, q}\right)$.

6. First-order necessary optimality conditions. We start the derivation of first-order conditions with the Fréchet-differentiability of the control-to-state operator $\mathcal{S}$ (cf. Definition 4.2) in section 6.1, which is one of the crucial points of the first-order analysis for $(\mathrm{P})$. However, using the analysis for the linearized equation, presented in section 5 , the implicit function theorem yields the desired differentiability of $\mathcal{S}$ as well as of the Lagrange function, which is defined in a standard way; see Definition 6.4 below. Later, in sections 6.2 and 6.3 , we reformulate the derivative of the Lagrange function by introducing an adjoint PDE system which leads to first-order necessary optimality conditions in the form of a Karush-Kuhn-Tucker (KKT)-type optimality system.

For the subsequent analysis we redefine $S$ as $S:=] 0, T[$. Recall that the state space is given by $Y=W^{1, r}\left(S ; H_{\Omega}^{-1, q}\right) \cap L^{r}\left(S ; H^{1, q}\right)$ with $r$ and $s$ as defined in Definition 4.1.

Definition 6.1. We define

$$
y(u):=\left(\begin{array}{c}
\zeta(u) \\
\varphi(u)
\end{array}\right) \in Y \times L^{\infty}\left(S ; H_{D}^{1, q}\right),
$$

where $\zeta(u)$ and $\varphi(u)$ are the solutions of (3.9) and (3.10) associated with $u$. Moreover, let $\mathcal{A}: Y \times L^{\infty}\left(S ; H_{D}^{1, q}\right) \rightarrow L^{r}\left(S ; H_{\Omega}^{-1, q}\right) \times L^{\infty}\left(S ; H_{D}^{-1, q}\right)$ be defined by

$$
\mathcal{A}(y):=\left(\begin{array}{c}
\partial_{t} \zeta+K \zeta-\left(\sigma\left(\zeta+\iota_{0}\right) \nabla \varphi\right) \cdot \nabla \varphi \\
-\nabla \cdot \sigma\left(\zeta+\iota_{0}\right) \nabla \varphi
\end{array}\right)
$$

Hence (1.1)-(1.7) is equivalent to

$$
\mathcal{A}(y)=\left(\begin{array}{c}
\tilde{\alpha} \\
\mathcal{I} u
\end{array}\right)
$$

Copyright (c) by SIAM. Unauthorized reproduction of this article is prohibited. 
where $\mathcal{I}: L^{\infty}\left(S ; L^{2}\left(\Gamma_{N}\right)\right) \rightarrow L^{\infty}\left(S ; H_{D}^{-1, q}(\Omega)\right)$ is defined by $\tilde{u}=\mathcal{I} u($ cf. Remark 3.2). Therefore, in view of Theorem 3.5, (6.1) admits a unique solution for every $u \in$ $L^{\infty}(] 0, T\left[; L^{2}\left(\Gamma_{N}\right)\right)$.

6.1. Differentiability of the control-to-state mapping. As stated above, we will utilize the implicit function theorem to prove the Fréchet-differentiability of $\mathcal{S}$. To this end let us introduce the mapping $\mathcal{T}: Y \times L^{\infty}\left(S ; L^{2}(\Gamma)\right) \rightarrow L^{r}\left(S ; H_{\Omega}^{-1, q}\right) \times$ $L^{\infty}\left(S ; H_{D}^{-1, q}\right)$,

$$
\mathcal{T}(y, u):=\mathcal{A}(y)-\left(\begin{array}{c}
\alpha \\
\mathcal{I} u
\end{array}\right)
$$

and hence (6.1) is equivalent to $\mathcal{T}(y, u)=0$.

TheOREM 6.2. The control-to-state operator $\mathcal{S}$ is continuously Fréchet-differentiable from $L^{\infty}\left(S ; L^{2}\left(\Gamma_{N}\right)\right)$ to $Y \times L^{\infty}\left(S ; H_{D}^{1, q}\right)$. Its derivative at the point $u$ in the direction $h \in L^{\infty}\left(S ; L^{2}\left(\Gamma_{N}\right)\right)$ is given by the solution of

$$
\begin{array}{rlrl}
\partial_{t} \theta^{\prime}-\operatorname{div}\left(\kappa \nabla \theta^{\prime}\right) & =\left(\sigma^{\prime}(\theta) \theta^{\prime} \nabla \varphi\right) \cdot \nabla \varphi+2(\sigma(\theta) \nabla \varphi) \cdot \nabla \varphi^{\prime} & \text { in } Q, \\
\nu \cdot \kappa \nabla \theta^{\prime}+\alpha \theta^{\prime} & =0 \quad \text { on } \Sigma, & & \\
\theta^{\prime}(0) & =0 \quad \text { in } \Omega, & & \\
-\operatorname{div}\left(\sigma(\theta) \nabla \varphi^{\prime}\right) & =\operatorname{div}\left(\sigma^{\prime}(\theta) \theta^{\prime} \nabla \varphi\right) & & \text { in } Q, \\
\nu \cdot \sigma(\theta) \nabla \varphi^{\prime} & =-\nu \cdot \sigma^{\prime}(\theta) \theta^{\prime} \nabla \varphi+h & & \text { on } \Sigma_{N}, \\
\nu \cdot \sigma(\theta) \nabla \varphi^{\prime} & =-\nu \cdot \sigma^{\prime}(\theta) \theta^{\prime} \nabla \varphi & & \text { on } \left.\left(\partial \Omega \backslash \bar{\Gamma}_{N} \cup \Gamma_{D}\right) \times\right] 0, T[, \\
\varphi^{\prime} & =0 & & \text { on } \Sigma_{D},
\end{array}
$$

where $(\theta, \varphi)=\mathcal{S}(u)$ and $\left(\theta^{\prime}, \varphi^{\prime}\right) \in Y \times L^{\infty}\left(S ; H_{D}^{1, q}\right)$ is a solution in the sense of Definition 5.2.

Proof. We apply the implicit function theorem to $\mathcal{T}(y, u)$ to verify the assertion. First, Theorem 3.5 implies that, for every $u \in L^{\infty}\left(S ; L^{2}\left(\Gamma_{N}\right)\right)$, there is a $y(u) \in Y \times L^{\infty}\left(S ; H_{D}^{1, q}\right)$ such that $\mathcal{T}(y(u), u)=0$. Next, we show that $\mathcal{T}$ is continuously Fréchet-differentiable with respect to $y$ from $Y \times L^{\infty}\left(S ; H_{D}^{1, q}\right)$ to $L^{r}\left(S ; H_{\Omega}^{-1, q}\right) \times$ $L^{\infty}\left(S ; H_{D}^{-1, q}\right)$. The Nemytskii-operator associated with $\sigma$ is Fréchet-differentiable in $L^{\infty}\left(S ; L^{\infty}\left(\Omega ; \mathcal{B}\left(\mathbb{R}^{2}\right)\right)\right)$ because of Assumption 5.1(iv), and thus, thanks to the continuous embedding, also from $W^{1, r}\left(S ; H_{\Omega}^{-1, q}\right) \cap L^{r}\left(S ; H^{1, q}\right)$ to $L^{\infty}\left(S ; L^{\infty}\left(\Omega ; \mathcal{B}\left(\mathbb{R}^{2}\right)\right)\right)$. Furthermore, the Nemytskii-operator $\Phi: L^{\infty}\left(S ; L^{q}\right) \rightarrow L^{\infty}\left(S ; L^{q / 2}\right)$, defined by

$$
\Phi(v)(x, t):=v(x, t)^{2},
$$

is clearly continuously Fréchet-differentiable from $L^{\infty}\left(S ; L^{q}\right)$ to $L^{\infty}\left(S ; L^{q / 2}\right)$. Consequently, the chain rule implies the continuous Fréchet-differentiability of $|\nabla \varphi|^{2}$ from $L^{\infty}\left(S ; H_{D}^{1, q}\right)$ to $L^{\infty}\left(S ; L^{q / 2}\right)$. Since all other constituents of $\mathcal{T}$ are linear and bounded in their respective functions spaces, this gives the continuous Fréchet-differentiability of $\mathcal{T}$.

It remains to verify that $\partial_{y} \mathcal{T}(y, u)$ is continuously invertible. Given an arbitrary $g=\left(g_{1}, g_{2}\right) \in L^{r}\left(S ; H_{\Omega}^{-1, q}\right) \times L^{\infty}\left(S ; H_{D}^{-1, q}\right)$, the equation $\partial_{y} \mathcal{T}(y, u) y^{\prime}=g$ is equivalent to

$$
\begin{aligned}
\partial_{t} \zeta^{\prime}+K \zeta^{\prime} & =\left(\sigma^{\prime}\left(\zeta+\iota_{0}\right) \zeta^{\prime} \nabla \varphi\right) \cdot \nabla \varphi+2\left(\sigma\left(\zeta+\iota_{0}\right) \nabla \varphi\right) \cdot \nabla \varphi^{\prime}+g_{1}, \\
-\nabla \cdot\left(\sigma\left(\zeta+\iota_{0}\right) \nabla \varphi^{\prime}\right) & =\nabla \cdot\left(\sigma^{\prime}\left(\zeta+\iota_{0}\right) \zeta^{\prime} \nabla \varphi\right)+g_{2},
\end{aligned}
$$

Copyright $@$ ㅇ by SIAM. Unauthorized reproduction of this article is prohibited. 
with $y^{\prime}=\left(\zeta^{\prime}, \varphi^{\prime}\right)$. We observe that it coincides with (5.10) and (5.11) with $\iota_{0}^{\prime}=0$, which of course corresponds to $\theta_{0}^{\prime}=0$. Hence, Theorem 5.3 yields the unique existence of $y^{\prime}$ in $Y \times L^{\infty}\left(S ; H_{D}^{1, q}\right)$ (cf. Remark 5.6), giving in turn the invertibility of $\partial_{y} \mathcal{T}(y, u)$. Therefore, the implicit function theorem implies that $y(u)$ is as smooth as $\mathcal{T}$ and thus continuously Fréchet-differentiable. The particular form of $\mathcal{S}^{\prime}$ immediately follows from

$$
y^{\prime}(u) h=-\partial_{y} \mathcal{T}(y(u), u)^{-1} \partial_{u} \mathcal{T}(y(u), u) h=\partial_{y} \mathcal{T}(y(u), u)^{-1}\left(\begin{array}{c}
0 \\
\mathcal{I} h
\end{array}\right) .
$$

Notice that $\mathcal{I}$ is linear and continuous and consequently Fréchet-differentiable.

Remark 6.3. Based on Theorem 5.3, system (6.2)-(6.8) is also uniquely solvable if the inhomogeneity is only an element of $L^{s}\left(S ; H_{D}^{-1, q}\right)$. The associated solution is also denoted by $\theta^{\prime}$ and $\varphi^{\prime}$; i.e.,

$$
\left(\theta^{\prime}, \varphi^{\prime}\right) \in W_{0}^{1, s}(] 0, T\left[; H_{\Omega}^{-1, q}\right) \cap L^{s}(] 0, T\left[; H^{1, q}\right) \times L^{s}(] 0, T\left[; H_{D}^{1, q}\right)
$$

solves

$$
\begin{aligned}
\partial_{t} \theta^{\prime}+K \theta^{\prime} & =\left(\sigma^{\prime}(\theta) \theta^{\prime} \nabla \varphi\right) \cdot \nabla \varphi+2(\sigma(\theta) \nabla \varphi) \cdot \nabla \varphi^{\prime}, \\
-\nabla \cdot\left(\sigma(\theta) \nabla \varphi^{\prime}\right) & =\nabla \cdot\left(\sigma^{\prime}(\theta) \theta^{\prime} \nabla \varphi\right)+\tilde{h} .
\end{aligned}
$$

Notice, however, that the above proof cannot be carried out with this notion of solutions to (6.2)-(6.8) since the Nemytskii-operator $\Phi$ is clearly not Fréchet-differentiable from $L^{s}\left(S ; L^{q}\right)$ to $L^{s}\left(S ; L^{q / 2}\right)$.

It is well known that the Lagrange multipliers associated with pointwise state constraints are in general only regular Borel measures; see, for instance, Casas [5]. Hence we define the Lagrange function associated with $(\mathrm{P})$ as follows.

DeFINITION 6.4. The space of regular Borel measures on $\bar{Q}$ is denoted by $\mathcal{M}(\bar{Q})$. The Lagrange function $\mathfrak{L}: L^{\infty}\left(S ; L^{2}\left(\Gamma_{N}\right)\right) \times \mathcal{M}(\bar{Q}) \rightarrow \mathbb{R}$ associated with $(\mathrm{P})$ is given by

$$
\mathfrak{L}(u, \mu)=j(u)+\left\langle\mathcal{S}_{1}(u)-\theta_{\max }, \mu\right\rangle_{C(\bar{Q}), \mathcal{M}(\bar{Q})},
$$

where $j$ is the reduced objective functional defined in Definition 4.2.

Remark 6.5. Notice that $\mathfrak{L}$ is well defined since, by the Riesz representation theorem, $\mathcal{M}(\bar{Q})$ can be identified with the dual space of $C(\bar{Q})$ and since Theorem 3.5(iv) implies that $\mathcal{S}_{1}(u)=\theta(u) \in C(\bar{Q})$.

Corollary 6.6. By the chain rule $\mathfrak{L}$ is continuously Fréchet-differentiable w.r.t. $u$ from $L^{\infty}\left(S ; L^{2}\left(\Gamma_{N}\right)\right)$ to $\mathbb{R}$, and its derivative at $u \in L^{\infty}\left(S ; L^{2}\left(\Gamma_{N}\right)\right)$ in direction $h \in L^{\infty}\left(S ; L^{2}\left(\Gamma_{N}\right)\right)$ is given by

$$
\partial_{u} \mathfrak{L}(u, \mu) h=\int_{D}\left(\theta(T)-\theta_{d}\right) \theta^{\prime}(T) d x+\beta \int_{\Sigma_{N}} u h d s d t+\left\langle\theta^{\prime}, \mu\right\rangle_{C(\bar{Q}), \mathcal{M}(\bar{Q})}
$$

with $\theta^{\prime}=\mathcal{S}_{1}^{\prime}(u) h=\left\langle(1,0)^{\top}, \mathcal{S}^{\prime}(u) h\right\rangle_{\mathbb{R}^{2}}$; i.e., $y^{\prime}:=\left(\theta^{\prime}, \varphi^{\prime}\right)=\mathcal{S}^{\prime}(u) h$ is the solution of $(6.2)-(6.8)$.

In the next section, we will reformulate the derivative of $\mathfrak{L}$ by introducing an adjoint state which is a solution of a PDE system, adjoint to (5.1)-(5.6), with measures on the right-hand side.

Copyright $@$ by SIAM. Unauthorized reproduction of this article is prohibited. 
6.2. An adjoint equation involving measures. In the subsequent section, we discuss the following equation, which is the system formally adjoint to (5.1)-(5.6):

$$
\begin{aligned}
-\partial_{t} \vartheta-\operatorname{div}(\kappa \nabla \vartheta) & =\left(\sigma^{\prime}(\theta) \vartheta \nabla \varphi\right) \cdot \nabla \varphi-\left(\sigma^{\prime}(\theta) \nabla \varphi\right) \cdot \nabla \psi+f_{1} & & \text { in } Q, \\
\nu \cdot \kappa \nabla \vartheta+\alpha \vartheta & =f_{2} & & \text { on } \partial \Omega \times] 0, T[, \\
\vartheta(T) & =\vartheta_{T} & & \text { in } \Omega, \\
-\operatorname{div}(\sigma(\theta) \nabla \psi) & =-2 \operatorname{div}(\sigma(\theta) \vartheta \nabla \varphi)+g_{1} & & \text { in } Q, \\
\nu \cdot \sigma(\theta) \nabla \psi & =2 \nu \cdot \sigma(\theta) \vartheta \nabla \varphi+g_{2} & & \text { on } \left.\left(\partial \Omega \backslash \Gamma_{D}\right) \times\right] 0, T[, \\
\psi & =0 & & \text { on } \left.\Gamma_{D} \times\right] 0, T[.
\end{aligned}
$$

The regularity of the inhomogeneities $f_{1}, f_{2}, g_{1}$, and $g_{2}$ and of the terminal value $\vartheta_{T}$ will be specified in the subsequent analysis. The analysis for (6.12)-(6.17), carried out in the following, mainly relies on a duality argument in the spirit of Amann [2]; i.e., we use Theorem 5.3 to prove existence and uniqueness of solutions to (6.12)-(6.17).

Definition 6.7. Let $q$ and $s$ be real numbers that satisfy the conditions of Assumption 5.1, and denote their conjugate exponents by $q^{\prime}$ and $s^{\prime}$ such that

$$
\left.q^{\prime} \in\right] \max \left\{\tilde{q}_{0}^{\prime}, q_{1}^{\prime}\right\}, 2\left[\text { and } s^{\prime} \in\right] 1, q / 2[,
$$

where $\tilde{q}_{0}$ and $q_{1}$ are the numbers from Theorems 3.7 and 3.18 .

Definition 6.8. Let $q, s \in \mathbb{R}$ satisfy the conditions of Definition 6.7. Then, we set

$$
\begin{aligned}
W_{s^{\prime}} & :=W^{1, s^{\prime}}\left(S ; H_{\Omega}^{-1, q^{\prime}}\right) \cap L^{s^{\prime}}\left(S ; H^{1, q^{\prime}}\right), \\
W_{s, 0} & :=W_{0}^{1, s}\left(S ; H_{\Omega}^{-1, q}\right) \cap L^{s}\left(S ; H^{1, q}\right) .
\end{aligned}
$$

The associated dual spaces are denoted by the superscript $*$. Moreover, given $r, m>1$, we define

$$
H_{1 / r^{\prime}, r}^{1, m}:=\left(H_{\Omega}^{-1, m}, H^{1, m}\right)_{1-1 / r, r},
$$

where $r^{\prime}$ satisfies $1 / r^{\prime}=1-1 / r$.

Note that, due to the duality properties of real interpolation functors,

$$
\begin{aligned}
H_{1 / s, s^{\prime}}^{1, q^{\prime}} & =\left(H_{\Omega}^{-1, q^{\prime}}, H^{1, q^{\prime}}\right)_{1 / s, s^{\prime}} \\
& =\left(H^{1, q^{\prime}}, H_{\Omega}^{-1, q^{\prime}}\right)_{1 / s^{\prime}, s^{\prime}}=\left(\left(H_{\Omega}^{-1, q}, H^{1, q}\right)_{1 / s^{\prime}, s}\right)^{*}=\left(H_{1 / s^{\prime}, s}^{1, q}\right)^{*}
\end{aligned}
$$

holds true. Let us now define the notion of weak and strong solutions in the spirit of Amann [2].

Definition 6.9. Let $q$ and $s$ be numbers according to Definition 6.7. Suppose that the inhomogeneities $f_{1}$ and $f_{2}$ define an element $\tilde{f}$ of $L^{s^{\prime}}\left(S ; H_{\Omega}^{-1, q^{\prime}}\right)$, whereas $g_{1}$ and $g_{2}$ are identified with $\tilde{g} \in L^{s^{\prime}}\left(S ; H_{D}^{-1, q^{\prime}}\right)$. Furthermore, let $\vartheta_{T}$ be given in $H_{1 / s, s^{\prime}}^{1, q^{\prime}}$. Then, a pair $(\vartheta, \psi) \in W_{s^{\prime}} \times L^{s^{\prime}}(] 0, T\left[; H_{D}^{1, q^{\prime}}\right)$ is said to be a strong solution of (6.12)-(6.17) if it satisfies the following:

1. the operator equations

$$
\begin{aligned}
-\partial_{t} \vartheta+K^{*} \vartheta & =\left(\sigma^{\prime}(\theta) \vartheta \nabla \varphi\right) \cdot \nabla \varphi+\left(\sigma^{\prime}(\theta) \nabla \varphi\right) \cdot \nabla \psi+\tilde{f}, \\
\left(-\nabla \cdot(\sigma(\theta) \nabla)^{*} \psi\right. & =-2 \nabla \cdot(\sigma(\theta) \vartheta \nabla \varphi)+\tilde{g},
\end{aligned}
$$

Copyright $@$ by SIAM. Unauthorized reproduction of this article is prohibited. 
2. the terminal condition

$$
\vartheta(T)=\vartheta_{T}
$$

Clearly, since $\kappa$ and $\sigma(\theta)$ are symmetric, $K$ and $-\nabla \cdot \sigma(\theta) \nabla$ are formally selfadjoint such that $K^{*}: H^{1, q^{\prime}} \rightarrow H_{\Omega}^{-1, q^{\prime}}$ and $(-\nabla \cdot \sigma(\theta) \nabla)^{*}: H_{D}^{1, q^{\prime}} \rightarrow H_{D}^{-1, q^{\prime}}$ are defined analogously to (3.2) and (3.4), respectively. Notice, moreover, that

$$
W_{s^{\prime}} \hookrightarrow C\left(\bar{S},\left(H_{\Omega}^{-1, q^{\prime}}, H^{1, q^{\prime}}\right)_{1 / s, s^{\prime}}\right)=C\left(\bar{S}, H_{1 / s, s^{\prime}}^{1, q^{\prime}}\right)
$$

(cf. Remark 3.15(iii)) such that (6.21) is well defined.

Definition 6.10. Let $f_{1}$ and $f_{2}$ define an element $\tilde{f} \in W_{s, 0}^{*}$, while $g_{1}$ and $g_{2}$ are identified with $\tilde{g} \in L^{s^{\prime}}\left(S ; H_{D}^{-1, q^{\prime}}\right)$. Moreover, $\vartheta_{T}$ is given in $H_{1 / s, s^{\prime}}^{1, q^{\prime}}$ with $\gamma$ as defined in Assumption 6.7. Then functions $\vartheta \in L^{s^{\prime}}\left(S ; H^{1, q^{\prime}}\right)$ and $\psi \in L^{s^{\prime}}\left(S ; H_{D}^{1, q^{\prime}}\right)$ are said to be a weak solution of (6.12)-(6.17) if they fulfill

$$
\begin{gathered}
\int_{S}\left\langle\partial_{t} \Theta, \vartheta\right\rangle_{H_{\Omega}^{-1, q}, H_{\Omega}^{1, q^{\prime}}} d t+\int_{\Sigma} \alpha \Theta \vartheta d \omega d t \\
\quad+\int_{Q}\left(\kappa \nabla \Theta \cdot \nabla \vartheta-\left(\sigma^{\prime}(\theta) \nabla \varphi\right) \cdot \nabla \varphi \Theta \vartheta+\left(\sigma^{\prime}(\theta) \nabla \varphi\right) \cdot \nabla \psi \Theta\right) d x d t \\
=\langle\Theta, \tilde{f}\rangle_{W_{s, 0}, W_{s, 0}^{*}}+\left\langle\Theta(T), \vartheta_{T}\right\rangle_{H_{1 / s^{\prime}, s}^{1, q}, H_{1 / s, s^{\prime}}^{1, q^{\prime}}} \quad \forall \Theta \in W_{s, 0}, \\
\left(-\nabla \cdot(\sigma(\theta) \nabla)^{*} \psi=-2 \nabla \cdot(\sigma(\theta) \vartheta \nabla \varphi)+\tilde{g} .\right.
\end{gathered}
$$

Note that the terminal condition is implicitly incorporated into this definition via the term $\left\langle\Theta(T), \vartheta_{T}\right\rangle$, which is well defined because of $W_{s, 0} \hookrightarrow C\left(\bar{S}, H_{1 / s^{\prime}, s}^{1, q}\right)$ and (6.18) (cf. also [2, sect. 7]).

Remark 6.11. Since the set $\mathcal{D}:=C_{0}^{\infty}\left(\left[0, T\left[, C^{\infty}(\bar{\Omega})\right)\right.\right.$ is dense in $W_{s, 0},(6.23)$ can equivalently be formulated with $\mathcal{D}$ as a test space.

ThEOREM 6.12.

(i) Under Assumption 6.7, for every right-hand side $\tilde{f} \in W_{s, 0}^{*}, \tilde{g} \in L^{s^{\prime}}\left(S ; H_{D}^{-1, q^{\prime}}\right)$ and every $\vartheta_{T} \in H_{1 / s, s^{\prime}}^{1, q^{\prime}}$, there exists a unique weak solution to (6.12)-(6.17) in the sense of Definition 6.10.

(ii) If the $\tilde{f}$ is more regular, i.e., $\tilde{f} \in L^{s^{\prime}}\left(S ; H_{\Omega}^{-1, q^{\prime}}\right)$, then the weak solution is a strong solution according to Definition 6.9 .

Proof. We mainly follow the lines of [2]. Let us start with the derivative of the operator $\mathcal{A}$ as given in Definition 6.1. As shown in the proof of Theorem $6.2, \mathcal{A}$ is continuously Fréchet-differentiable from $Y \times L^{\infty}\left(S ; H_{D}^{1, q}\right)$ to $L^{r}\left(S ; H_{\Omega}^{-1, q}\right) \times L^{\infty}\left(S ; H_{D}^{-1, q}\right)$, and its derivative at $y:=(\theta, \varphi)$ in direction $w:=(\Theta, \Phi)$ is given by

$$
\mathcal{A}^{\prime}(y) w=\left(\begin{array}{c}
\partial_{t} \Theta+K \Theta-\left(\sigma^{\prime}(\theta) \Theta \nabla \varphi\right) \cdot \nabla \varphi-2(\sigma(\theta) \nabla \varphi) \cdot \nabla \Phi \\
-\nabla \cdot(\sigma(\theta) \nabla \Phi)-\nabla \cdot\left(\sigma^{\prime}(\theta) \Theta \nabla \varphi\right)
\end{array}\right) .
$$

In view of Theorem 5.3, $\mathcal{A}^{\prime}(y)$ is also well defined and, by the open mapping theorem, continuously invertible when considered as an operator from $W_{s, 0} \times L^{s}\left(S ; H_{D}^{1, q}\right)$ to $L^{s}\left(S ; H_{\Omega}^{-1, q}\right) \times L^{s}\left(S ; H_{D}^{-1, q}\right)$ (cf. also Remark 6.3). For simplicity let us denote this operator also by $\mathcal{A}^{\prime}(y)$. Now, set $p=(\vartheta, \psi) \in L^{s^{\prime}}\left(S ; H_{\Omega}^{1, q^{\prime}}\right) \times L^{s^{\prime}}\left(S ; H_{D}^{1, q^{\prime}}\right)$. Then 
the adjoint operator $\mathcal{A}^{\prime}(y)^{*}: L^{s^{\prime}}\left(S ; H^{1, q^{\prime}}\right) \times L^{s^{\prime}}\left(S ; H_{D}^{1, q^{\prime}}\right) \rightarrow W_{s, 0}^{*} \times L^{s^{\prime}}\left(S ; H_{D}^{-1, q^{\prime}}\right)$ is given by

$$
\begin{aligned}
& \left\langle\mathcal{A}^{\prime}(y)^{*} p, w\right\rangle=\left\langle p, \mathcal{A}^{\prime}(y) w\right\rangle \\
& =\int_{S}\left\langle\partial_{t} \Theta, \vartheta\right\rangle_{H_{\Omega}^{-1, q}, H^{1, q^{\prime}}} d t+\int_{\Sigma} \alpha \Theta \vartheta d \omega d t \\
& \quad+\int_{Q}\left(\kappa \nabla \Theta \cdot \nabla \vartheta-\left(\sigma^{\prime}(\theta) \nabla \varphi\right) \cdot \nabla \varphi \Theta \vartheta+\left(\sigma^{\prime}(\theta) \Theta \nabla \varphi\right) \cdot \nabla \psi\right) d x d t \\
& \quad+\int_{Q}((\sigma(\theta) \nabla \Phi) \cdot \nabla \psi-2(\sigma(\theta) \nabla \varphi) \cdot \nabla \Phi \vartheta) d x d t .
\end{aligned}
$$

As stated above, in view of the open mapping theorem, Theorem 5.3 implies that $\mathcal{A}^{\prime}(y)$ is continuously invertible, giving in turn

$$
\left(\mathcal{A}^{\prime}(y)^{*}\right)^{-1} \in \mathcal{B}\left(W_{s, 0}^{*} \times L^{s^{\prime}}\left(S ; H_{D}^{-1, q^{\prime}}\right), L^{s^{\prime}}\left(S ; H^{1, q^{\prime}}\right) \times L^{s^{\prime}}\left(S ; H_{D}^{1, q^{\prime}}\right)\right) .
$$

Hence, for every right-hand side $\tilde{b} \in W_{s, 0}^{*} \times L^{s^{\prime}}\left(S ; H_{D}^{-1, q^{\prime}}\right)$, there is a unique solution of the equation

$$
\left\langle\mathcal{A}^{\prime}(y)^{*} p, w\right\rangle=\langle\tilde{b}, w\rangle \quad \forall w \in W_{s, 0} \times L^{s}\left(S ; H_{D}^{1, q}\right)
$$

in $L^{s^{\prime}}\left(S ; H^{1, q^{\prime}}\right) \times L^{s^{\prime}}\left(S ; H_{D}^{1, q^{\prime}}\right)$. Now suppose that $\tilde{b}$ takes the form

$$
\langle\tilde{b}, w\rangle=\langle\Theta, \tilde{f}\rangle_{W_{s, 0}, W_{s, 0}^{*}}+\left\langle\Theta(T), \vartheta_{T}\right\rangle_{H_{1 / s^{\prime}, s}^{1, q}, H_{1 / s, s^{\prime}}^{1, q^{\prime}}}+\langle\Phi, \tilde{g}\rangle_{L^{s}\left(S ; H_{D}^{1, q}\right), L^{s^{\prime}}\left(S ; H_{D}^{-1, q^{\prime}}\right)},
$$

where $\vartheta_{T}$ clearly defines an element of $W_{s, 0}^{*}$ due to the above-mentioned embeddings. If one inserts this definition of $\tilde{b}$ and test functions $(\Theta, 0)$ and $(0, \Phi)$, respectively, with arbitrary $\Theta$ and $\Phi$, into (6.27), then the definition of $\mathcal{A}^{\prime}(y)^{*}$ in (6.26) immediately yields part (i) of the theorem.

Next assume that $\tilde{f}$ is more regular, i.e., $\tilde{f} \in L^{s^{\prime}}\left(S ; H_{\Omega}^{-1, q^{\prime}}\right)$, and insert $\Theta(x, t)=$ $z(t) v(x)$ with $z \in C_{0}^{\infty}[0, T]$ and $v \in H^{1, q}(\Omega)$ as test functions into (6.23) such that

$$
\begin{aligned}
& \left\langle\int_{S} \partial_{t} z \vartheta d t, v\right\rangle_{H_{\Omega}^{-1, q^{\prime}}, H^{1, q}} \\
& =\left\langle\int_{S}\left(-K^{*} \vartheta+\left(\sigma^{\prime}(\theta) \vartheta \nabla \varphi\right) \cdot \nabla \varphi+\left(\sigma^{\prime}(\theta) \nabla \varphi\right) \cdot \nabla \psi+\tilde{f}\right) z d t, v\right\rangle_{H_{\Omega}^{-1, q^{\prime}, H^{1, q}}} .
\end{aligned}
$$

Since $v$ was chosen arbitrary, we have for the distributional derivative of $\vartheta$

$$
\begin{aligned}
\partial_{t} \vartheta(z)=-\int_{S} \partial_{t} z \vartheta d t & \\
=-\int_{S}\left(-K^{*} \vartheta\right. & +\left(\sigma^{\prime}(\theta) \vartheta \nabla \varphi\right) \cdot \nabla \varphi \\
& \left.+\left(\sigma^{\prime}(\theta) \nabla \varphi\right) \cdot \nabla \psi+\tilde{f}\right) z d t \quad \forall z \in C_{0}^{\infty}[0, T] .
\end{aligned}
$$

Thus, $\partial_{t} \vartheta$ is a regular distribution generated by

$$
\mathfrak{f}:=K^{*} \vartheta-\left(\sigma^{\prime}(\theta) \vartheta \nabla \varphi\right) \cdot \nabla \varphi-\left(\sigma^{\prime}(\theta) \nabla \varphi\right) \cdot \nabla \psi-\tilde{f} .
$$

Copyright $@$ by SIAM. Unauthorized reproduction of this article is prohibited. 
Consequently, if we identify $\partial_{t} \vartheta$ with $\mathfrak{f},(6.19)$ is obtained. Moreover, this immediately implies that $\partial_{t} \vartheta$ is an element of $L^{s^{\prime}}\left(S, H_{\Omega}^{-1, q^{\prime}}\right)$ due to the regularity of $\mathfrak{f}$. Hence we obtain $\vartheta \in W_{s^{\prime}}$, i.e., the regularity of a strong solution. Thus, with regard to $[2$, Prop. 5.1], we are allowed to integrate by parts w.r.t. time and obtain

$$
\begin{aligned}
& \int_{S}\left\langle-\partial_{t} \vartheta+K^{*} \vartheta-\left(\sigma^{\prime}(\theta)\right.\right.\left.\nabla \varphi) \cdot \nabla \varphi-\left(\sigma^{\prime}(\theta) \nabla \varphi\right) \cdot \nabla \psi-\tilde{f}, \Theta\right\rangle_{H_{\Omega}^{-1, q^{\prime}}, H^{1, q}} d t \\
&+\left\langle\Theta(T), \vartheta(T)-\vartheta_{T}\right\rangle_{H_{1 / s^{\prime}, s}^{1, q}, H_{1 / s, s^{\prime}}^{1, q^{\prime}}}=0 \quad \forall \Theta \in W_{s, 0} .
\end{aligned}
$$

In view of (6.19), this finally gives the terminal condition (6.21).

Now, let $s>2 q /(q-2)$ such that

$$
H_{1 / s^{\prime}, s}^{1, q}=\left(H_{\Omega}^{-1, q}, H^{1, q}\right)_{1-1 / s, s} \hookrightarrow C(\bar{\Omega})
$$

(cf. Lemma 3.17(i) and (3.19)). In addition, Remark 3.15(iii) yields $W_{s, 0} \hookrightarrow C(\bar{Q})$. Since both embeddings are dense, we therefore have

$$
\mathcal{M}(\bar{\Omega}) \hookrightarrow H_{1 / s, s}^{1, q^{\prime}} \text { and } \mathcal{M}(\bar{Q}) \hookrightarrow W_{s, 0}^{*},
$$

provided that $s>2 q /(q-2)$. Here, $\mathcal{M}(\bar{\Omega})$ and $\mathcal{M}(\bar{Q})$ denote the spaces of regular measures on $\bar{\Omega}$ and $\bar{Q}$, respectively (see Definition 6.4). As in the case of $\mathcal{M}(\bar{Q}) \simeq$ $C(\bar{Q})^{*}$, we identify $\mathcal{M}(\bar{\Omega})$ with the dual of $C(\bar{\Omega})$ by the Riesz representation theorem. Consequently, Theorem 6.12 implies the following.

Corollary 6.13. Assume that $\mu \in \mathcal{M}(\bar{Q})$ is given and that the restriction of $\mu$ on $0 \times \bar{\Omega}$ is zero. Moreover, denote the restrictions of $\mu$ on $Q, \Sigma:=\partial \Omega \times] 0, T[$, and $T \times \bar{\Omega}$ by $\mu_{Q}, \mu_{\Sigma}$, and $\mu_{T}$. Then the system

$$
\begin{aligned}
-\partial_{t} \vartheta-\operatorname{div}(\kappa \nabla \vartheta) & =\left(\sigma^{\prime}(\theta) \vartheta \nabla \varphi\right) \cdot \nabla \varphi-\left(\sigma^{\prime}(\theta) \nabla \varphi\right) \cdot \nabla \psi+\mu_{Q} & & \text { in } Q, \\
\nu \cdot \kappa \nabla \vartheta+\alpha \vartheta & =\mu_{\Sigma} \quad & \text { on } \partial \Omega \times] 0, T[, & \\
\vartheta(T) & =\mu_{T} \quad & \text { in } \Omega, & \\
-\operatorname{div}(\sigma(\theta) \nabla \psi) & =-2 \operatorname{div}(\sigma(\theta) \vartheta \nabla \varphi) & & \text { in } Q, \\
\nu \cdot \sigma(\theta) \nabla \psi & =2 \nu \cdot \sigma(\theta) \vartheta \nabla \varphi & & \text { on } \left.\left(\partial \Omega \backslash \Gamma_{D}\right) \times\right] 0, T[, \\
\psi & =0 & & \text { on } \left.\Gamma_{D} \times\right] 0, T[
\end{aligned}
$$

admits a unique weak solution $(\vartheta, \psi) \in L^{s^{\prime}}\left(S ; H^{1, q^{\prime}}\right) \times L^{s^{\prime}}\left(S ; H_{D}^{1, q^{\prime}}\right), s^{\prime}<2 q /(q+2)$, in the sense of Definition 6.10.

Remark 6.14. We note that, if measures appear on the right-hand side of the adjoint equation, then $\vartheta \in L^{s^{\prime}}\left(S ; H^{1, q^{\prime}}\right)$ such that no weak differentiability of the adjoint state w.r.t. time can be expected in this case.

6.3. Derivation of the optimality system. Now we are in a position to state the first-order necessary optimality conditions for $(\mathrm{P})$. Let us begin with the notion of local optimality, as follows.

Definition 6.15. A function $\bar{u} \in L^{\infty}\left(S ; L^{2}\left(\Gamma_{N}\right)\right)$ is called locally optimal for (P) if there is an $\varepsilon>0$ such that $j(\bar{u}) \leq j(u)$ holds for all feasible $u \in L^{\infty}\left(S ;\left(\Gamma_{N}\right)\right)$ with $\|u-\bar{u}\|_{L^{\infty}\left(S ; L^{2}\left(\Gamma_{N}\right)\right)} \leq \varepsilon$.

Recall that the Lagrange function is Fréchet-differentiable w.r.t. $u$ by Corollary 6.6. Hence we continue with the definition of Lagrange multipliers associated with the state constraints in $(\mathrm{P})$.

Copyright $@$ by SIAM. Unauthorized reproduction of this article is prohibited. 
Definition 6.16. Let $\bar{u}$ be a locally optimal solution of $(\mathrm{P})$; then $\mu \in \mathcal{M}(\bar{Q})$ is said to be a Lagrange multiplier associated with the state constraints in $(\mathrm{P})$ if

$$
\begin{gathered}
\partial_{u} \mathfrak{L}(\bar{u}, \mu)(u-\bar{u}) \geq 0 \quad \forall u \in U_{a d}, \\
\mu \geq 0, \\
\left\langle\theta-\theta_{\max }, \mu\right\rangle_{C(\bar{Q}), \mathcal{M}(\bar{Q})}=0
\end{gathered}
$$

hold true.

Here, (6.35) is equivalent to

$$
\langle y, \mu\rangle_{C(\bar{Q}), \mathcal{M}(\bar{Q})} \geq 0 \quad \forall y \in\{y \in C(\bar{Q}) \mid y(x, t) \geq 0 \forall(x, t) \in \bar{Q}\} .
$$

Moreover, (6.36) is referred to as the complementary slackness conditions in all that follows. The following theorem states the first-order necessary optimality conditions for (P), i.e., the existence of Lagrange multipliers in the sense of Definition 6.16. It is, for instance, proven by Casas in [5].

THEOREM 6.17. Assume that $\bar{u}$ is a locally optimal solution of $(\mathrm{P})$ and satisfies the following linearized Slater condition: There exist an interior point $u_{0} \in U_{\text {ad }}$ and a real number $\delta>0$ such that

$$
\mathcal{S}_{1}(\bar{u})(x, t)+\mathcal{S}_{1}^{\prime}(\bar{u})\left(u_{0}-\bar{u}\right)(x, t) \leq \theta_{\max }(x, t)-\delta \quad \forall(x, t) \in \bar{Q} .
$$

Then, there exists a Lagrange multiplier $\mu \in \mathcal{M}(\bar{Q})$ according to Definition 6.16 such that (6.34)-(6.36) are satisfied.

It is well known that a certain constraint qualification is needed to ensure the existence of Lagrange multipliers, such as the linearized Slater condition (6.38) (cf. also Zowe and Kurcyusz [32]). Notice that this condition requires us to consider the state constraints in $C(\bar{Q})$. The Lagrange multipliers are elements of the associated dual space and therefore in general only regular Borel measures, i.e., $\mu \in \mathcal{M}(\bar{Q})$. Next, let us transform (6.34)-(6.36) into the optimality system of (P) by introducing the adjoint state. To that end, let us consider a fixed but arbitrary local optimum $\bar{u}$ with associated state $\bar{y}=(\bar{\theta}, \bar{\varphi})$. Moreover, we again denote the derivative of $\mathcal{S}$ in an arbitrary direction $h \in L^{\infty}\left(S ; L^{2}(\Gamma)\right)$ by $y^{\prime}$; i.e., $y^{\prime}=\mathcal{S}^{\prime}(\bar{u}) h$. Now, consider $h$ as an element of $L^{s}\left(S ; H_{D}^{-1, q}\right)$ with $q$ and $s$ according to Definition 6.7, i.e., $\left.q \in\right] 2$, $\min \left\{\tilde{q}_{0}, q_{1}\right\}[$ and $\left.s \in] q /(q-2), \infty\right]$. Then $y^{\prime}$ clearly also solves

$$
\mathcal{A}^{\prime}(\bar{y}) y^{\prime}=\left(\begin{array}{l}
0 \\
h
\end{array}\right)
$$

where, as in the proof of Theorem $6.12, \mathcal{A}^{\prime}(\bar{y})$ is considered as an operator from $W_{s, 0} \times$ $L^{s}\left(S ; H_{D}^{1, q}\right)$ to $L^{s}\left(S ; H_{\Omega}^{-1, q}\right) \times L^{s}\left(S ; H_{D}^{-1, q}\right)$ (which is well defined and continuously invertible; cf. also Remark 6.3). Note that, according to Definition 6.8, $W_{s, 0}$ is given by $W_{s, 0}=W_{0}^{1, s}\left(S ; H_{\Omega}^{-1, q}\right) \cap L^{s}\left(S ; H^{1, q}\right)$. Now, define $p_{1}=\left(\vartheta_{1}, \psi_{1}\right)$ as the solution of

$$
\begin{aligned}
-\partial_{t} \vartheta_{1}-\operatorname{div}\left(\kappa \nabla \vartheta_{1}\right) & =\left(\sigma^{\prime}(\bar{\theta}) \vartheta_{1} \nabla \bar{\varphi}\right) \cdot \nabla \varphi-\left(\sigma^{\prime}(\bar{\theta}) \nabla \varphi\right) \cdot \nabla \psi_{1} & & \text { in } Q, \\
\nu \cdot \kappa \nabla \vartheta_{1}+\alpha \vartheta_{1} & =0 \quad \text { on } \partial \Omega \times] 0, T[, & & \\
\vartheta_{1}(T) & =\mathcal{I}_{D}\left(\chi_{D} \bar{\theta}(T)-\theta_{d}\right) & & \text { in } \Omega, \\
-\operatorname{div}\left(\sigma(\bar{\theta}) \nabla \psi_{1}\right) & =-2 \operatorname{div}\left(\sigma(\bar{\theta}) \vartheta_{1} \nabla \bar{\varphi}\right) & & \text { in } Q, \\
\nu \cdot \sigma(\bar{\theta}) \nabla \psi_{1} & =2 \nu \cdot \sigma(\bar{\theta}) \vartheta_{1} \nabla \bar{\varphi} & & \text { on } \left.\left(\partial \Omega \backslash \Gamma_{D}\right) \times\right] 0, T[, \\
\psi & =0 & & \text { on } \left.\Gamma_{D} \times\right] 0, T[,
\end{aligned}
$$

Copyright (c) by SIAM. Unauthorized reproduction of this article is prohibited. 
where $\mathcal{I}_{D}: L^{2}(D) \rightarrow H_{1 / s, s^{\prime}}^{1, q^{\prime}}(\Omega)$ is defined by

$$
\left\langle\mathcal{I}_{D}(g), \Theta\right\rangle_{H_{1 / s, s^{\prime}}^{1, q^{\prime}}, H_{1 / s^{\prime}, s}^{1, q}}:=\int_{D} g \Theta d x, \quad g \in L^{2}(D), \Theta \in H_{1 / s^{\prime}, s}^{1, q},
$$

while $\chi_{D}$ is the characteristic function on $D$. Notice that $\mathcal{I}_{D}$ is well defined since

$$
H_{1 / s^{\prime}, s}^{1, q}=\left(H_{\Omega}^{-1, q}, H^{1, q}\right)_{1-1 / s, s} \hookrightarrow H^{2 \eta-1, q}
$$

with $\eta=2 / q<1-1 / s$ because of $s>q /(q-2)$ such that $2 \eta-1>0$ due to $q<4$. Therefore $H_{1 / s^{\prime}, s}^{1, q} \hookrightarrow L^{2}(D)$. Theorem 6.12 implies that there is a strong solution $p_{1} \in W_{s^{\prime}} \times L^{s^{\prime}}\left(S ; H_{D}^{1, q^{\prime}}\right)$ to $(6.40)-(6.45)$ that satisfies

$$
\left\langle\mathcal{A}(\bar{y})^{*} p_{1}, w\right\rangle=\int_{D}\left(\bar{\theta}(T)-\theta_{d}\right) \Theta(T) d x
$$

for all $w=(\Theta, \Phi) \in W_{s, 0} \times L^{s}\left(S ; H_{D}^{1, q}\right)$. Next, assume $\left.\left.s \in\right] 2 q /(q-2), \infty\right]$, and introduce $p_{2}=\left(\vartheta_{2}, \psi_{2}\right) \in L^{s^{\prime}}\left(S ; H^{1, q^{\prime}}\right) \times L^{s^{\prime}}\left(S ; H_{D}^{1, q^{\prime}}\right)$ as a weak solution of (6.28)(6.33), where the inhomogeneity $\mu$ is the Lagrange multiplier associated with the state constraints in $(\mathrm{P})$. Notice in this context that, due to Assumption 2.4(iii), the state constraint is not active at $t=0$. Consequently, the positivity of the Lagrange multipliers and the complementary slackness conditions yield that the restriction of $\mu$ on $0 \times \bar{\Omega}$ is indeed zero, as in case of (6.28)-(6.33). Hence, $p_{2}$ solves

$$
\left\langle\mathcal{A}^{\prime}(y)^{*} p_{2}, w\right\rangle=\langle\Theta, \mu\rangle_{C(\bar{Q}), \mathcal{M}(\bar{Q})} \quad \forall w \in W_{s, 0} \times L^{s}\left(S ; H_{D}^{1, q}\right)
$$

with $s>2 q /(q-2)$ (such that $W_{s, 0} \hookrightarrow C(\bar{Q})$; cf. Corollary 6.13). Thus, taking this together with (6.39), we obtain

$$
\begin{aligned}
\int_{D}\left(\bar{\theta}(T)-\theta_{d}\right) & \theta^{\prime}(T) d x+\left\langle\theta^{\prime}, \mu\right\rangle_{C(\bar{Q}), \mathcal{M}(\bar{Q})} \\
& =\left\langle\mathcal{A}^{\prime}(\bar{y})^{*} p_{1}, y^{\prime}\right\rangle+\left\langle\mathcal{A}^{\prime}(\bar{y})^{*} p_{2}, y^{\prime}\right\rangle=\left\langle p_{1}+p_{2}, \mathcal{A}^{\prime}(\bar{y}) y^{\prime}\right\rangle \\
& =\left\langle p_{1}+p_{2},\left(\begin{array}{c}
0 \\
h
\end{array}\right)\right\rangle=\int_{\Sigma}\left(\psi_{1}+\psi_{2}\right) h d s d t .
\end{aligned}
$$

Inserting this into (6.11) and (6.34) and a pointwise evaluation of the arising inequality imply by standard arguments

$$
\bar{u}(x, t)=\Pi_{a d}\left\{-\frac{1}{\beta}\left(\tau_{N} \psi_{1}+\tau_{N} \psi_{2}\right)\right\},
$$

where $\Pi_{a d}$ denotes the pointwise projection operator on $U_{a d}$ and $\tau_{N}$ is again the trace operator on $\Gamma_{N}$. In this way, we have proven the following result stating the first-order necessary conditions for $(\mathrm{P})$.

TheOrem 6.18. Let $\bar{u} \in L^{\infty}\left(S ; L^{2}\left(\Gamma_{N}\right)\right)$ be a local optimum of $(\mathrm{P})$ with associated state

$$
\bar{y}=(\bar{\theta}, \bar{\varphi}) \in W_{0}^{1, r}\left(S ; H_{\Omega}^{-1, q}\right) \cap L^{r}\left(S ; H^{1, q}\right) \times L^{\infty}\left(S ; H_{D}^{1, q}\right)
$$

with $\left.q \in] 2, \min \left\{\tilde{q}_{0}, q_{1}\right\}\right]$ and $r>2 q /(q-2)$. Suppose further that a function $u_{0} \in U_{a d}$ exists such that the linearized Slater condition (6.38) is fulfilled. Then there exist a

Copyright (c) by SIAM. Unauthorized reproduction of this article is prohibited. 
Lagrange multiplier $\mu \in \mathcal{M}(\bar{Q})$ and adjoint states

$$
\begin{aligned}
& p_{1}=\left(\vartheta_{1}, \psi_{1}\right) \in W^{1, s_{1}^{\prime}}(] 0, T\left[; H_{\Omega}^{-1, q^{\prime}}\right) \cap L^{s_{1}^{\prime}}(] 0, T\left[; H^{1, q^{\prime}}\right), \\
& p_{2}=\left(\vartheta_{2}, \psi_{2}\right) \in L^{s_{2}^{\prime}}\left(S ; H^{1, q^{\prime}}\right) \times L^{s_{2}^{\prime}}\left(S ; H_{D}^{1, q^{\prime}}\right),
\end{aligned}
$$

with $q^{\prime}=q /(q-1), s_{1}^{\prime}<q / 2$, and $s_{2}^{\prime}<2 q /(q+2)$, such that the following conditions are satisfied:

- the state system (1.1)-(1.7) in the sense of Definition 3.3,

- the first adjoint system (6.40)-(6.45) in the sense of Definition 6.9,

- the second adjoint system (6.28)-(6.33) in the sense of Definition 6.10,

- the positivity property (6.37) of the multipliers,

- the complementary slackness conditions (6.36),

- the projection formula (6.46).

Notice that, due to the trace theorem, $\Pi_{a d}$ clearly maps $L^{s^{\prime}}\left(S ; H^{1-1 / q^{\prime}, q^{\prime}}\left(\Gamma_{N}\right)\right)$ into $L^{s^{\prime}}\left(S ; H^{1-1 / q^{\prime}, q^{\prime}}\left(\Gamma_{N}\right)\right)$ such that the generic regularity for a local optimal control is given by

$$
\bar{u} \in L^{\infty}\left(S ; L^{2}\left(\Gamma_{N}\right)\right) \cap L^{s^{\prime}}\left(S ; H^{1-1 / q^{\prime}, q^{\prime}}\left(\Gamma_{N}\right)\right)
$$

with $s^{\prime}<2 q /(q+2), q \leq q_{\max }=\min \left\{\tilde{q}_{0}, q_{1}\right\}<4$ (see Remark 3.8), such that $s^{\prime}<4 / 3$. In addition, we have $q^{\prime} \in\left[q_{\min }^{\prime}, 2\left[\right.\right.$, where $q_{\min }^{\prime}$ is the conjugate exponent to $q_{\max }$. Note further that the optimality system can be simplified by introducing $p=p_{1}+p_{2}$ as an adjoint state, i.e., the weak solution of

$$
\begin{aligned}
-\partial_{t} \vartheta-\operatorname{div}(\kappa \nabla \vartheta) & =\left(\sigma^{\prime}(\bar{\theta}) \vartheta \nabla \bar{\varphi}\right) \cdot \nabla \varphi-\left(\sigma^{\prime}(\bar{\theta}) \nabla \varphi\right) \cdot \nabla \psi+\mu_{Q} & & \text { in } Q, \\
\nu \cdot \kappa \nabla \vartheta+\alpha \vartheta & \left.=\mu_{\Sigma} \quad \text { on } \partial \Omega \times\right] 0, T[, & & \\
\vartheta(T) & =\mathcal{I}_{D}\left(\chi_{D} \bar{\theta}(T)-\theta_{d}\right)+\mu_{T} & & \text { in } \Omega, \\
-\operatorname{div}(\sigma(\bar{\theta}) \nabla \psi) & =-2 \operatorname{div}(\sigma(\bar{\theta}) \vartheta \nabla \varphi) & & \text { in } Q, \\
\nu \cdot \sigma(\bar{\theta}) \nabla \psi & =2 \nu \cdot \sigma(\bar{\theta}) \vartheta \nabla \bar{\varphi} & & \text { on } \left.\left(\partial \Omega \backslash \Gamma_{D}\right) \times\right] 0, T[, \\
\psi & =0 & & \text { on } \left.\Gamma_{D} \times\right] 0, T[.
\end{aligned}
$$

7. A specific application and numerical tests. As mentioned in the introduction, a problem of type (P) arises, for instance, when optimizing the heat treatment of steel by means of an electric current. This procedure is applied in the automotive industry for the hardening of gear racks as part of the widely used rack-and-pinion steering mechanism. Here the workpiece is heated up by direct current and then rapidly cooled down by means of water nozzles to produce a hard martensitic outer layer. For a detailed description of such heating procedures, see [25]. The aim of the optimization is a uniform heating of the teeth of the gear rack which is essential for the hardening process in order to avoid thermal stress and to guarantee a uniform hardening of the tooth system. Thus the measurement domain $D$ in the objective functional of $(\mathrm{P})$ is the domain which is covered by the teeth of the gear rack. Since it is essential to prevent melting during the hardening process, the bound $\theta_{\max }$ in the state constraint of $(\mathrm{P})$ is given by the melting temperature of the material. In addition, the control constraints in $(\mathrm{P})$ reflect the maximum electrical power that can be induced into the workpiece.

In the following, we report on two numerical tests for this particular application problem. The respective optimality system, described in Theorem 6.18 , is solved by means of a projected gradient method, fitting to the first-order analysis presented in 


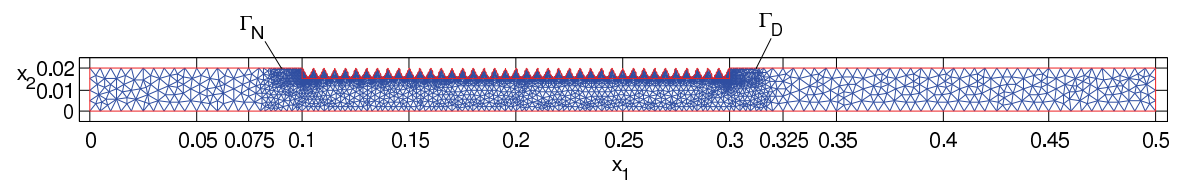

FIG. 7.1. Computational domain.

TABLE 7.1

Material parameters within the numerical tests.

\begin{tabular}{ccccccc}
\hline$\varrho$ & $C_{p}$ & $\kappa$ & $\alpha$ & $\theta_{0}$ & $\theta_{l}$ & $\theta_{d}$ \\
\hline \hline $7900 \frac{\mathrm{kg}}{\mathrm{m}^{3}}$ & $470 \frac{\mathrm{J}}{\mathrm{kg} \mathrm{K}}$ & $50 \frac{\mathrm{W}}{\mathrm{mK}}$ & $20 \frac{\mathrm{W}}{\mathrm{m}^{2} \mathrm{~K}}$ & $290 \mathrm{~K}$ & $290 \mathrm{~K}$ & $1500 \mathrm{~K}$ \\
\hline
\end{tabular}

the preceding sections. While the control constraints are incorporated into the projected gradient method, the pointwise state constraints are regularized by means of a quadratic penalization; see [21] and the references therein. Moreover, the PDEs arising in each step of the optimization algorithm are discretized by linear finite elements in combination with a semi-implicit time stepping. Furthermore, the control is discretized by a piecewise linear and continuous spatial ansatz function, while piecewise constant ansatz functions are used in time.

For the computational domain we choose the two-dimensional simplified gear rack shown in Figure 7.1. Aside from $\sigma$, the material parameters are constant and chosen to approximate the realistic distributions. The particular values used are shown in Table 7.1. Here $C_{p}$ and $\varrho$ refer to the specific heat capacity and the density, respectively, that enter the heat equation via

$$
C_{p} \varrho \partial_{t} \theta-\operatorname{div}(\kappa \nabla \theta)=(\sigma(\theta) \nabla \varphi) \cdot \nabla \varphi,
$$

which clearly does not influence the theory since they are assumed to be constant. Notice that all parameters are positive constants such that the hypotheses in Assumptions 2.3, 2.4, and 5.1 are satisfied. Moreover, in this case, the function $\sigma$ is a scalar-valued function, depending only on $\theta$, i.e., $\sigma: \mathbb{R} \rightarrow \mathbb{R}$, which is given by

$$
\sigma(\theta)=\left(a+b \theta+c \theta^{2}+d \theta^{3}\right)^{-1} \quad \text { if } \theta \in[0,10000],
$$

with $a=4.9659 \cdot 10^{-7}, b=8.4121 \cdot 10^{-10}, c=-3.7246 \cdot 10^{-13}$, and $d=6.1960$. $10^{-17}$ (see [11] for details). On $\mathbb{R} \backslash[0,10000], \sigma$ is smoothly extended such that $0<\sigma_{0} \leq \sigma(\theta) \leq \sigma_{1}<\infty$ is satisfied for all $\theta \in \mathbb{R}$. Hence it fulfills the conditions in Assumptions 2.3 and 5.1. Finally, the end time $T$ was set to $2.0 \mathrm{~s}$. The Tikhonov parameter $\beta$ within the objective functional is set to $10^{-13}$ to compensate for the comparatively high values of the control (see below).

In the following, two numerical tests are presented, differing concerning the inequality constraints in $(\mathrm{P})$. While there are inequality constraints only on the control but not on the state in the first example, we choose $\theta_{\max }=1800 \mathrm{~K}$ in the second test case. In both cases we set $u_{\max }=7 \cdot 10^{7} \mathrm{~A} / \mathrm{m}^{2}$. Note that both test cases are covered by the above theory, since the control is uniformly bounded in $L^{\infty}(] 0, T\left[; L^{2}\left(\Gamma_{N}\right)\right)$. In all of what follows we refer to the first example as free optimization, since no state constraints are present in this case. It serves as a reference problem in comparison to the state-constrained case. Figure 7.2 shows a detail of the tooth-system at end time 


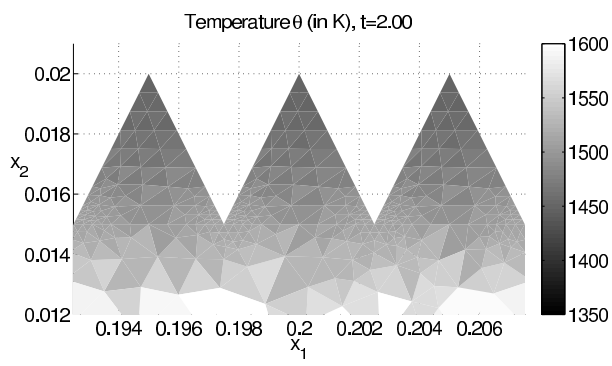

FIG. 7.2. Temperature at end time for the free optimization.

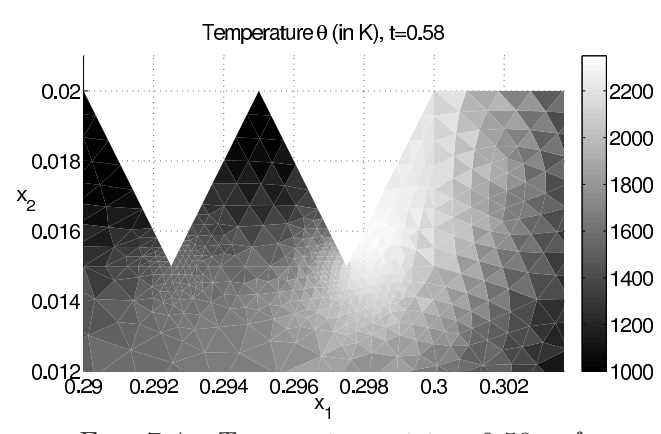

Fig. 7.4. Temperature at $t=0.58 \mathrm{~s}$ for the free optimization.

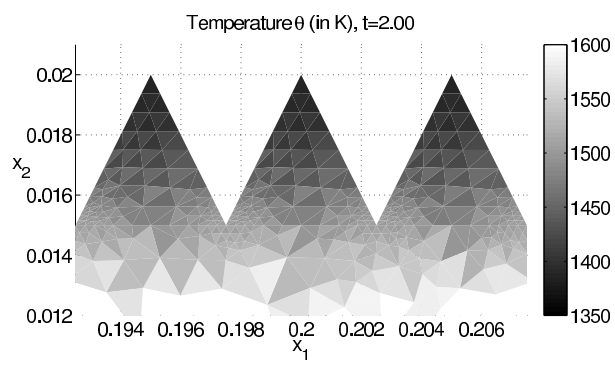

FIG. 7.3. Temperature at end time for the state-constrained optimization.

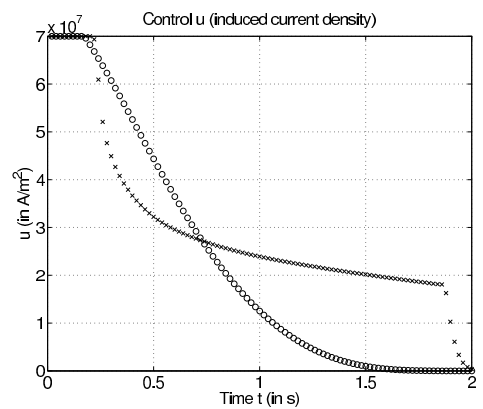

FIG. 7.5. Time evolution of the control in case of free (circles) and state-constrained (crosses) optimization.

for this case. We observe that the desired temperature of $1500 \mathrm{~K}$ is nearly reached. However, since no state constraints are imposed, the material is in danger of melting in the corners of the tooth-system, as Figure 7.4 illustrates. The situation changes if the temperature is forced to stay below the melting temperature by the additional state constraints in $(\mathrm{P})$. In the second numerical test this is approximately enforced by a quadratic penalty term, resulting in a maximum temperature of $1805.1 \mathrm{~K}$ in the right corner of the tooth-system at $t=0.26 \mathrm{~s}$. However, in this case, the temperature distribution differs more significantly from the desired $1500 \mathrm{~K}$ than does the free optimization, as Figure 7.3 shows. This observation appears natural since the inequality constraints on the state do not allow for the extreme temperature evolution observed in the case of the free optimization. Therefore, it seems that a time interval of $2.0 \mathrm{~s}$ is not sufficient for heating up the workpiece to $1500 \mathrm{~K}$ before cooling it down if, at the same time, melting should be prevented.

In Figure 7.5 the time evolution of the control $u$ in case of free and stateconstrained optimization is depicted. Here the crosses refer to the state-constrained case, while the circles represent the free optimization. The values are taken at a fixed but arbitrary point on $\Gamma_{N}$. One observes that the time evolution of the control differs significantly between the cases. Moreover, the control significantly decreases in time in both examples. An explanation for this observation is the fact that the current does not flow directly through the teeth. Only the area straight below the tooth system is heated up intensely by the current. Afterwards, heat conduction from this area into the teeth increases the temperature in the tooth system. Thus, to achieve a temperature distribution in the teeth as uniform as possible, it appears reasonable to heat up the area below the teeth comparatively fast to ensure a uniform heat conduction into the teeth. Finally the optimal potential $\varphi$ in the state-constrained case at end time 


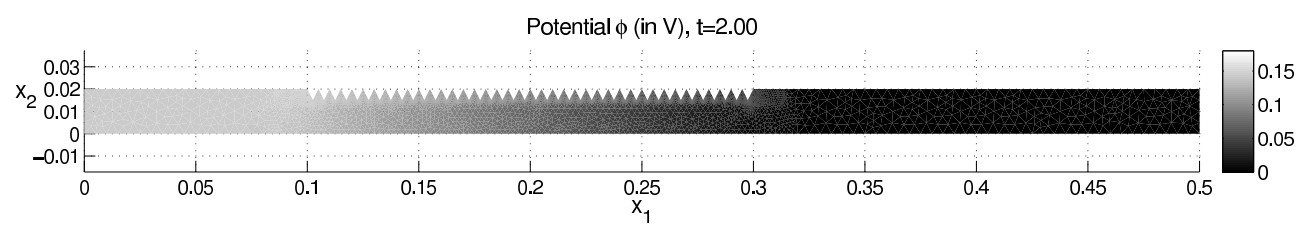

FIG. 7.6. Potential at end time for the state-constrained optimization.

$T=2.0 \mathrm{~s}$ is shown in Figure 7.6. We observe that the expected decay from cathode to anode is reflected by the numerical computations.

Appendix. Here, the basic properties of solutions to parabolic equations, mentioned at the beginning of section 3.1, are proven. We start with Lemma 3.13.

Proof of Lemma 3.13. (i) Based on [26, Chap. 2.5], we estimate for $s, t \in\left[T_{0}, T_{1}\right]$ with $s<t$

$$
\begin{aligned}
\left\|e^{-s A} x-e^{-t A} x\right\|_{\mathcal{D}} & =\left\|A e^{-s A} x-A e^{-t A} x\right\|_{X} \\
& \leq\left\|A e^{-\frac{s}{2} A}\right\|_{\mathcal{B}(X)}\left\|e^{-\frac{s}{2} A} x-e^{-(t-s) A} e^{-\frac{s}{2} A} x\right\|_{X} \\
& \leq c\left\|A e^{-\frac{s}{2} A}\right\|_{\mathcal{B}(X)}^{2}\|x\|_{X}|t-s|,
\end{aligned}
$$

which gives the first assertion.

(ii) First one notices that $\left\|e^{-t A}\right\|_{\mathcal{B}(\mathcal{D})} \leq\left\|e^{-t A}\right\|_{\mathcal{B}(X)}$, which implies by interpolation that $\left\|e^{-t A}\right\|_{\mathcal{B}\left([X, \mathcal{D}]_{\tau}\right)} \leq\left\|e^{-t A}\right\|_{\mathcal{B}(X)}$ for all $t \in[0, \infty[$ and all $\tau \in] 0,1[$ (see [31, Chap. 1.2.2 and 1.9.3]). Now let $T>0$ be given and $s, t \in] 0, T[$; then we have by the reiteration theorem (see [31, Chap. 1.9.3])

$$
\begin{aligned}
& \frac{\left\|e^{-t A} x-e^{-s A} x\right\|_{[X, \mathcal{D}]_{\rho}}}{|t-s|^{\tau-\rho}} \leq c \frac{\left\|e^{-t A} x-e^{-s A} x\right\|_{\left[X,[X, \mathcal{D}]_{\tau}\right]_{\frac{\rho}{\tau}}}}{|t-s|^{\tau\left(1-\frac{\rho}{\tau}\right)}} \\
& \leq c\left(\frac{\left\|e^{-t A} x-e^{-s A} x\right\|_{X}}{|t-s|^{\tau}}\right)^{\left(1-\frac{\rho}{\tau}\right)}\left\|e^{-t A} x-e^{-s A} x\right\|_{[X, \mathcal{D}]_{\tau}}^{\frac{\rho}{\tau}} \\
& \leq c\left(\sup _{s \in] 0, T[}\left\|e^{-s A}\right\|_{\mathcal{B}(X)}\right)^{\left(1-\frac{\rho}{\tau}\right)} \\
& \left(\sup _{t \in] 0, T[} \frac{\left\|e^{-t A} x-x\right\|_{X}}{|t|^{\tau}}\right)^{\left(1-\frac{\rho}{\tau}\right)}\left(2 \sup _{s \in] 0, T[}\left\|e^{-s A}\right\|_{\mathcal{B}\left([X, \mathcal{D}]_{\tau}\right)}\right)^{\frac{\rho}{\tau}}\|x\|_{[X, \mathcal{D}]_{\tau}}^{\frac{\rho}{\tau}} \\
& \leq 2 c\left(\sup _{s \in] 0, T[}\left\|e^{-s A}\right\|_{\mathcal{B}(X)}\right)\left(\sup _{t \in] 0, T[} \frac{\left\|e^{-t A} x-x\right\|_{X}}{|t|^{\tau}}\right)^{\left(1-\frac{\rho}{\tau}\right)}\|x\|_{[X, \mathcal{D}]_{\tau}}^{\frac{\rho}{\tau}} .
\end{aligned}
$$

By a well-known theorem (see [31, Chap. 1.13.2] or [24, Prop. 2.2.4 and Rem. 2.2.5]), one has

$$
\sup _{t \in] 0, T[} \frac{\left\|e^{-t A} x-x\right\|_{X}}{|t|^{\tau}} \leq c_{1}\|x\|_{(X, \mathcal{D})_{\tau, \infty}}
$$

with a positive constant $c_{1}$ (independent from $x \in[X, \mathcal{D}]_{\tau}$ ) and, secondly, the continuous embedding $[X, \mathcal{D}]_{\tau} \hookrightarrow(X, \mathcal{D})_{\tau, \infty}$; see [31, Chap. 1.10.3]. Hence, we continue (A.1) by

$$
\frac{\left\|e^{-t A} x-e^{-s A} x\right\|_{[X, \mathcal{D}]_{\rho}}}{|t-s|^{\tau-\rho}} \leq 2 c\left(\sup _{s \in] 0, T[}\left\|e^{-s A}\right\|_{\mathcal{B}(X)}\right)\|x\|_{[X, \mathcal{D}]_{\tau}},
$$

which proves (ii) (see also [1, Chap. II.5.3]). 
Proof of Lemma 3.16. (i) During this proof, let $S, S^{\prime}$ denote the intervals $] T_{0}, T_{1}$ [ and $] T_{0}, T^{\prime}$, respectively, with $T^{\prime}<T_{1}$. Associate with any function $f \in L^{r}\left(S^{\prime} ; X\right)$ a function $\mathcal{I} f$ which is defined as follows:

$$
\mathcal{I} f(t):=\left\{\begin{array}{l}
0 \quad \text { if } t \in] T_{0}, T_{1}-T^{\prime}[, \\
\left.f\left(t-\left(T_{1}-T^{\prime}\right)\right) \text { if } t \in\right] T_{1}-T^{\prime}, T_{1}[.
\end{array}\right.
$$

Clearly, $\mathcal{I}$ then provides isometric injections

$$
\begin{aligned}
L^{r}\left(S^{\prime} ; X\right) & \rightarrow L^{r}(S ; X), \\
W_{0}^{1, r}\left(S^{\prime} ; X\right) \cap L^{r}\left(S^{\prime} ; \mathcal{D}\right) & \rightarrow W_{0}^{1, r}(S ; X) \cap L^{r}(S ; \mathcal{D}), \\
C_{0}\left(\bar{S}^{\prime} ;(X, \mathcal{D})_{1-\frac{1}{r}, r}\right) & \rightarrow C_{0}\left(\bar{S} ;(X, \mathcal{D})_{1-\frac{1}{r}, r}\right)
\end{aligned}
$$

If we indicate $\mathcal{L}$ by its interval end and write $\mathcal{L}_{T_{1}}$ and $\mathcal{L}_{T^{\prime}}$, then we have for any $f \in L^{r}\left(S^{\prime} ; X\right)$ the identity $\mathcal{L}_{T_{1}} \mathcal{I} f=\mathcal{I L}_{T^{\prime}} f$. Thus, one may estimate for any $f \in$ $L^{r}\left(S^{\prime} ; X\right)$

$$
\begin{aligned}
\left\|\mathcal{L}_{T^{\prime}} f\right\|_{W_{0}^{1, r}\left(S^{\prime} ; X\right) \cap L^{r}\left(S^{\prime} ; \mathcal{D}\right)} & =\left\|\mathcal{I} \mathcal{L}_{T^{\prime}} f\right\|_{W_{0}^{1, r}(S ; X) \cap L^{r}(S ; \mathcal{D})}=\left\|\mathcal{L}_{T_{1}} \mathcal{I} f\right\|_{W_{0}^{1, r}(S ; X) \cap L^{r}(S ; \mathcal{D})} \\
& \leq\left\|\mathcal{L}_{T_{1}}\right\|\|\mathcal{I} f\|_{L^{r}(S ; X)}=\left\|\mathcal{L}_{T_{1}}\right\|\|f\|_{L^{r}\left(S^{\prime} ; X\right)},
\end{aligned}
$$

which implies $\left\|\mathcal{L}_{T^{\prime}}\right\| \leq\left\|\mathcal{L}_{T_{1}}\right\|$.

(ii) Denoting the embedding constant of

$$
W_{0}^{1, r}(S ; X) \cap L^{r}(S ; \mathcal{D}) \hookrightarrow C_{0}\left(\bar{S} ;(X, \mathcal{D})_{1-\frac{1}{r}, r}\right)
$$

by $c_{T}$, we estimate

$$
\begin{aligned}
\|w\|_{C_{0}\left(\bar{S}_{1} ;(X, \mathcal{D})_{1-\frac{1}{r}, r}\right)} & =\|\mathcal{I} w\|_{C_{0}\left(\bar{S} ;(X, \mathcal{D})_{1-\frac{1}{r}, r}\right)} \\
& \leq c_{T}\|\mathcal{I} w\|_{W_{0}^{1, r}(S ; X) \cap L^{r}(S ; \mathcal{D})}=c_{T}\|w\|_{W_{0}^{1, r}\left(S_{1} ; X\right) \cap L^{r}\left(S_{1} ; \mathcal{D}\right)}
\end{aligned}
$$

Thus, the embedding constant which corresponds to the interval $] T_{0}, T^{\prime}$ [ is at most $c_{T}$.

Proof of Lemma 3.17. (i) is obtained from well-known embedding theorems; see [31, Chap. 1.3.3 and 1.10].

(ii) Obviously, the norm of $\mathcal{E}_{C}$ does not depend on the interval length. This, combined with the Lemma 3.16, gives the assertion.

(iii) First, the estimate

$$
\begin{aligned}
\left\|w(t)-w\left(t_{0}\right)\right\|_{X} & =\left\|\int_{t_{0}}^{t} w^{\prime}(s) d s\right\| \leq\left(\int_{t_{0}}^{t}\left\|w^{\prime}(s)\right\|^{r} d s \|\right)^{\frac{1}{r}}\left(\int_{t_{0}}^{t} d s\right)^{\frac{r-1}{r}} \\
& \leq\|w\|_{W^{1, r}(S ; X)}\left|t-t_{0}\right|^{\frac{r-1}{r}}
\end{aligned}
$$

implies a continuous embedding from $W^{1, r}(S ; X)$ into $C^{\frac{r-1}{r}}(S ; X)$. Let $\eta$ be a number from $] \tau, 1-\frac{1}{r}\left[\right.$. Then, by setting $\delta=\frac{r-1}{r}$ and $\lambda=\frac{\tau}{\eta}$, we obtain by the reiteration 
theorem for complex interpolation (see [31, Chap. 1.9.3])

$$
\begin{aligned}
\frac{\|w(t)-w(s)\|_{[X, D]_{\tau}}}{|t-s|^{\delta(1-\lambda)}} & \leq c \frac{\|w(t)-w(s)\|_{\left[X,[X, D]_{\eta}\right]_{\lambda}}}{|t-s|^{\delta(1-\lambda)}} \\
& \leq c \frac{\|w(t)-w(s)\|_{X}^{1-\lambda}}{|t-s|^{\delta(1-\lambda)}}\|w(t)-w(s)\|_{[X, D]_{\eta}}^{\lambda} \\
& \leq c\left(\frac{\|w(t)-w(s)\|_{X}}{|t-s|^{\delta}}\right)^{1-\lambda}\left(2\|w\|_{C\left(\bar{S} ;[X, D]_{\eta}\right)}\right)^{\lambda} \\
& \leq c\|w\|_{W^{1, r}(S ; X) \cap L^{r}(S ; \mathcal{D})},
\end{aligned}
$$

which completes the proof.

Proof of Lemma 3.20. (i) The $L^{\infty}$ estimate follows from the fact that $K$ generates a contraction semigroup on $L^{\infty}$; see [15]. Further, $K$ generates an analytic semigroup on $H^{-1, q}$ (see [12] or [19]), and 0 belongs to its resolvent set because the resolvent is compact and 0 cannot be an eigenvalue due to Assumption 2.3; see [13, Lem. 1.36]. Hence, the Lipschitz continuity follows from Lemma 3.13(i).

(ii) Assume $\vartheta \in] \frac{2}{q}, \varsigma[$. We have for $\lambda=\varsigma$ and $\lambda=\vartheta$ the interpolation identity

$$
H^{\lambda, q}=\left[H_{\Omega}^{-1, q}, H^{1, q}\right]_{\frac{\lambda+1}{2}} ;
$$

see [14, Thm. 3.5]. Thus, the supposition $\theta_{0} \in H^{\varsigma, q}(\Omega)$ and Lemma 3.13(ii) imply $\left.\iota\right|_{] 0, T[} \in C^{\frac{s-\vartheta}{2}}(] 0, T\left[; H^{\vartheta, q}\right)$. An application of the (continuous) embedding $H^{\vartheta, q}(\Omega) \hookrightarrow$ $C^{\vartheta-\frac{2}{q}}(\Omega)$ (see [31, Chap. 4.6.1]) then proves the assertion.

\section{REFERENCES}

[1] H. Amann, Linear and Quasilinear Parabolic Problems, Birkhäuser, Basel, 1995.

[2] H. AmAnN, Nonautonomous parabolic equations involving measures, J. Math. Sci., 130 (2005), pp. $4780-4802$.

[3] S. N. Antontsev And M. Сhipot, The thermistor problem: Existence, smoothness, uniqueness, blowup, SIAM J. Math. Anal., 25 (1994), pp. 1128-1156.

[4] F. Bonnans And E. CASAS, An extension of Pontryagin's principle for state-constrained optimal control of semilinear elliptic equations and variational inequalities, SIAM J. Control Optim., 33 (1995), pp. 274-298.

[5] E. CASAS, Boundary control of semilinear elliptic equations with pointwise state constraints, SIAM J. Control Optim., 31 (1993), pp. 993-1006.

[6] E. Casas, J. C. De los Reyes, and F. Tröltzsch, Sufficient second-order optimality conditions for semilinear control problems with pointwise state constraints, SIAM J. Optim., 19 (2008), pp. 616-643.

[7] E. Casas And F. TrÖltzsch, Second-order necessary optimality conditions for some stateconstrained control problems of semilinear elliptic equations, Appl. Math. Optim., 39 (1999), pp. 211-227.

[8] E. Casas and J. Yong, Maximum principle for state-constrained control problems governed by quasilinear elliptic equations, Differential Integral Equations, 8 (1995), pp. 1-18.

[9] P. G. Ciarlet, The Finite Element Method for Elliptic Problems, Stud. Math. Appl. 4, NorthHolland, Amsterdam, 1979.

[10] G. CimatTi, Optimal control for the thermistor problem with a current limiting device, IMA J. Math. Control Inform., 24 (2007), pp. 339-345.

[11] S. Clain, J. Rappaz, M. Swierkosz, and R. Touzani, Numerical modeling of induction heating for two-dimensional geometries, Math. Models Methods Appl. Sci., 3 (1993), pp. 271-281.

[12] G. DoRe, $L^{p}$ regularity for abstract differential equations, in Proceedings of the International Conference in Memory of K. Yosida, Kyoto University, Japan, 1991, H. Komatsu, ed., Lecture Notes in Math. 1540, Springer, Berlin, 1993, pp. 25-38.

[13] H. Gajewski, K. Gröger, and K. Zacharias, Nichtlineare Operatorgleichungen und Operatordifferentialgleichungen, Akademie-Verlag, Berlin, 1974.

Copyright $@$ by SIAM. Unauthorized reproduction of this article is prohibited. 
[14] J. A. Griepentrog, K. Gröger, H. C. Kaiser, And J. Rehberg, Interpolation for function spaces related to mixed boundary value problems, Math. Nachr., 241 (2002), pp. 110-120.

[15] J. A. Griepentrog, H. C. Kaiser, and J. Rehberg, Heat kernel and resolvent properties for second order elliptic differential operators with general boundary conditions on $L^{p}$, Adv. Math. Sci. Appl., 11 (2001), pp. 87-112.

[16] P. Grisvard, Elliptic Problems in Nonsmooth Domains, Pitman, Boston, 1985.

[17] K. Gröger, A $W^{1, p}$-estimate for solutions to mixed boundary value problems for second order elliptic differential equations, Math. Ann., 283 (1989), pp. 679-687.

[18] K. GRÖGER, $W^{1, p}$-estimates of solutions to evolution equations corresponding to nonsmooth second order elliptic differential operators, Nonlinear Anal., 18 (1992), pp. 569-577.

[19] K. Gröger And J. RehberG, Resolvent estimates in $W^{-1, p}$ for second order elliptic differential operators in case of mixed boundary conditions, Math. Ann., 285 (1989), pp. 105-113.

[20] R. Haller-Dintelmann, C. Meyer, J. Rehberg, and A. Schiela, Hoelder continuity and optimal control for nonsmooth elliptic problems, Appl. Math. Optim., 60 (2009), pp. 397428.

[21] M. Hintermüller And K. Kunisch, Feasible and noninterior path-following in constrained minimization with low multiplier regularity, SIAM J. Control Optim., 45 (2006), pp. 11981221.

[22] V. Hrynkiv, S. Lenhart, and V. Protopopescu, Optimal control of convective boundary condition in a thermistor problem, SIAM J. Control Optim., 47 (2008), pp. 20-39.

[23] H.-C. Lee And T. Shilkin, Analysis of optimal control problems for the two-dimensional thermistor system, SIAM J. Control Optim., 44 (2005), pp. 268-282.

[24] A. Lunardi, Analytic Semigroups and Optimal Regularity in Parabolic Problems, Birkhäuser, Basel, 1995.

[25] A. Mühlbauer, Industrielle Elektrowärmetechnik, Vulkan-Verlag, Essen, 1992 (in German).

[26] A. PAZY, Semigroups of Linear Operators and Applications to Partial Differential Equations, Springer, Berlin, 1983.

[27] J.-P. RAYmond And F. TrÖLtzSch, Second order sufficient optimality conditions for nonlinear parabolic control problems with state constraints, Discrete Contin. Dynam. Systems, 6 (2000), pp. 431-450.

[28] M. R. Sidi Ammi, Optimal control for a nonlocal parabolic problem resulting from thermistor system, Int. J. Ecolog. Econ. Statist., 9 (2007), pp. 116-122.

[29] J. Simon, Compact sets in the space $L^{p}(0, T ; B)$, Ann. Mat. Pura Appl., 146 (1986), pp. 65-96.

[30] P. E. SobolevskiI, Coerciveness inequalities for abstract parabolic equations, Soviet. Mat. (Dokl.), 5 (1964), pp. 894-897.

[31] H. TRIebel, Interpolation Theory, Function Spaces, Differential Operators, North-Holland, Amsterdam, 1978.

[32] J. Zowe And S. KurCyusz, Regularity and stability for the mathematical programming problem in Banach spaces, Appl. Math. Optim., 5 (1979), pp. 49-62.

Copyright (c) by SIAM. Unauthorized reproduction of this article is prohibited. 